*For correspondence: cmeiklejohn2@unl.edu (CDM); daven.presgraves@rochester.edu (DCP)

Present address: ${ }^{\dagger}$ Department of Integrative Biology, University of California, Berkeley, Berkeley, United States; ${ }^{\ddagger}$ Department of Molecular Biology and Genetics, Field of Genetics, Genomics, and Development, Cornell University, New York, United States; ${ }^{\S}$ Pacific Biosciences of California, California, United States; \#Department of Organismic and Evolutionary Biology, Harvard University, Cambridge, United States; "Department of Developmental Biology, SloanKettering Institute, New York, United States; ${ }^{* \star}$ AncestryDNA, San Francisco, United States

Competing interests: The authors declare that no competing interests exist.

Funding: See page 22

Received: 28 January 2018 Accepted: 15 November 2018 Published: 13 December 2018

Reviewing editor: Molly Przeworski, Columbia University, United States

(c) Copyright Meiklejohn et al. This article is distributed under the terms of the Creative Commons Attribution License, which permits unrestricted use and redistribution provided that the original author and source are credited.

\title{
Gene flow mediates the role of sex chromosome meiotic drive during complex speciation
}

\section{Colin D Meiklejohn ${ }^{1 *}$, Emily L Landeen ${ }^{2 \dagger}$, Kathleen E Gordon ${ }^{1 \ddagger}$, Thomas Rzatkiewicz ${ }^{2}$, Sarah B Kingan ${ }^{2 \S}$, Anthony J Geneva"\#, Jeffrey P Vedanayagam ${ }^{2 \pi}$, Christina A Muirhead ${ }^{2}$, Daniel Garrigan ${ }^{2 * *}$, David L Stern ${ }^{3}$, Daven C Presgraves ${ }^{2 *}$}

${ }^{1}$ School of Biological Sciences, University of Nebraska, Lincoln, United States; ${ }^{2}$ Department of Biology, University of Rochester, New York, United States; ${ }^{3}$ Janelia Research Campus, Howard Hughes Medical Institute, Virginia, United States

Abstract During speciation, sex chromosomes often accumulate interspecific genetic incompatibilities faster than the rest of the genome. The drive theory posits that sex chromosomes are susceptible to recurrent bouts of meiotic drive and suppression, causing the evolutionary buildup of divergent cryptic sex-linked drive systems and, incidentally, genetic incompatibilities. To assess the role of drive during speciation, we combine high-resolution genetic mapping of $X$-linked hybrid male sterility with population genomics analyses of divergence and recent gene flow between the fruitfly species, Drosophila mauritiana and $D$. simulans. Our findings reveal a high density of genetic incompatibilities and a corresponding dearth of gene flow on the $X$ chromosome. Surprisingly, we find that a known drive element recently migrated between species and, rather than contributing to interspecific divergence, caused a strong reduction in local sequence divergence, undermining the evolution of hybrid sterility. Gene flow can therefore mediate the effects of selfish genetic elements during speciation.

DOl: https://doi.org/10.7554/eLife.35468.001

\section{Introduction}

Speciation involves the evolution of reproductive incompatibilities between diverging populations, including prezygotic incompatibilities that prevent the formation of hybrids and postzygotic incompatibilities that render hybrids sterile or inviable. Two patterns characterizing speciation implicate a special role for sex chromosomes in the evolution of postzygotic incompatibilities: Haldane's rule, the observation that hybrids of the heterogametic sex preferentially suffer sterility and inviability (Haldane, 1922; Wu and Davis, 1993; Orr, 1997; Laurie, 1997; Price and Bouvier, 2002; Presgraves, 2002; Coyne and Orr, 2004); and the large X-effect, the observation that the $X$ chromosome has a disproportionately large effect on hybrid sterility (Coyne and Orr, 1989; Coyne, 1992a; Presgraves, 2008). These patterns hold across a wide range of taxa, including female heterogametic (ZW) birds and Lepidoptera and male heterogametic (XY) plants, Drosophila, and mammals (Coyne and Orr, 1989; Coyne and Orr, 2004). We now know that these 'two rules of speciation' (Coyne and Orr, 1989) are, in part, attributable to the rapid evolution of genetic factors that cause interspecific hybrid sterility on the X chromosome relative to the autosomes (Tao and Hartl, 2003; Moehring et al., 2007; Masly and Presgraves, 2007; Presgraves, 2008; Good et al., 2008). The relatively rapid accumulation of $\mathrm{X}$-linked hybrid sterility factors is associated with reduced interspecific gene flow at X-linked versus autosomal loci (reviewed in Presgraves, 2018). Overall, these 
patterns show that, for many taxa with heteromorphic sex chromosomes, the $\mathrm{X}$ chromosome plays a large and fundamental role in speciation.

Given the taxonomic breadth of Haldane's rule, the large X-effect, and reduced interspecific gene flow on the $X$, understanding why the $X$ chromosome accumulates hybrid incompatibilities faster than the rest of the genome is imperative. At least five explanations have been proposed: faster X evolution (Charlesworth et al., 1987), gene traffic (Moyle et al., 2010), disrupted sex chromosome regulation in the germline (Lifschytz and Lindsley, 1972), the evolutionary origination of incompatibilities in parapatry (Höllinger and Hermisson, 2017), and meiotic drive (Hurst and Pomiankowski, 1991; Frank, 1991). Here, we focus on the potential role of meiotic drive. The drive theory posits that sex chromosomes are more susceptible than autosomes to invasion by selfish meiotic drive (sensu lato) elements (Hurst and Pomiankowski, 1991; Frank, 1991). Sex-linked drive compromises fertility and distorts sex ratios, which leads to evolutionary arms races between drivers, unlinked suppressors, and linked enhancers (Lindholm et al., 2016; Presgraves, 2008; Meiklejohn and Tao, 2010). These arms races can contribute to the evolution of hybrid male sterility, in at least two ways. Normally-suppressed drive elements might be aberrantly expressed in the naive genetic backgrounds of species hybrids, causing sterility rather than sex ratio distortion (Hurst and Pomiankowski, 1991; Frank, 1991). Alternatively, recurrent bouts of invasion, spread, and coevolution among drive, suppressor, and enhancer loci might cause interspecific divergence at these loci that incidentally cause hybrid sterility and map disproportionately to sex chromosomes (Presgraves, 2008; Meiklejohn and Tao, 2010).

Multiple lines of evidence support the plausibility of the drive theory. First, theoretical considerations and empirical evidence suggests that both active and suppressed sex chromosome meiotic drive systems are widespread in natural populations (Jaenike, 2001). Indeed, in one species, Drosophila simulans, three cryptic (normally suppressed) sex-ratio drive systems-Winters, Durham, and Paris-have been identified, involving distinct sets of $\mathrm{X}$-linked drive loci and autosomal and/or Y-linked suppressors (Tao et al., 2001; Tao et al., 2007a; Tao et al., 2007b; Helleu et al., 2016). Second, loci involved in cryptic sex-ratio systems co-localize with hybrid male sterility loci in genetic mapping experiments (Tao et al., 2001; Zhang et al., 2015; Orr and Irving, 2005). Third, at least one of the two X-linked hybrid sterility genes identified to date also causes meiotic drive (Phadnis and Orr, 2009). These discoveries confirm that recurrent bouts of drive and suppression have occurred and that cryptic drive genes can cause hybrid sterility. While these findings put the plausibility of the drive hypothesis beyond doubt, the question of its generality remains: what fraction of X-linked hybrid sterility factors evolved as a consequence of drive? We can furthermore ask whether, and how often, drive can impede the evolution of hybrid incompatibilities. The drive hypothesis assumes, for instance, that populations evolve in strict allopatry (simple speciation) and/ or that drive elements require particular population-specific genetic backgrounds for their activity. But for populations that diverge with some level of gene flow (complex speciation), drive elements can in principle migrate between species, thereby reducing divergence and potentially undermining the evolution of hybrid sterility (Macaya-Sanz et al., 2011; Crespi and Nosil, 2013; Seehausen et al., 2014).

Here, we investigate the special role of sex chromosomes in speciation with genetic mapping and population genomic analyses between Drosophila mauritiana and D. simulans. The human commensal species, D. simulans, originated on Madagascar, diverging from the sub-Saharan African species, D. melanogaster, 3 Mya (Lachaise et al., 1988; Dean and Ballard, 2004; Baudry et al., 2006; Kopp, 2006; Ballard, 2004). The island-endemic species, D. mauritiana, originated on the Indian Ocean island of Mauritius, diverging from D. simulans $\sim 240$ kya (Kliman et al., 2000; McDermott and Kliman, 2008; Garrigan et al., 2012). The two species are now isolated by geography-D. simulans has never been collected on Mauritius (David et alı, 1989)—and by multiple incomplete reproductive incompatibilities, including asymmetric premating isolation (Coyne, 1992b), postmating-prezygotic isolation (Price, 1997), and intrinsic postzygotic isolation ( $F_{1}$ hybrid males are sterile, $F_{1}$ hybrid females are fertile; Lachaise et al., 1986). Despite geographic and reproductive isolation, there is clear evidence for historical gene flow between the two species (Solignac and Monnerot, 1986; Solignac et al., 1986; Garrigan et al., 2012; Ballard, 2000a; Ballard, 2000b; Satta et al., 1988; Satta and Takahata, 1990). The X chromosome shows both an excess of factors causing hybrid male sterility (True et al., 1996b; Tao et al., 2003) and, correspondingly, a dearth of historical interspecific introgression (Garrigan et al., 2012). The rapid 
accumulation of $\mathrm{X}$-linked hybrid male sterility factors may have contributed to reduced $\mathrm{X}$-linked gene flow, limiting exchangeability at sterility factors and genetically linked loci (Muirhead and Presgraves, 2016).

To begin to assess the role of drive in the evolution of X-linked hybrid male sterility between these two species, we performed genetic mapping experiments using genotype-by-sequencing of advanced-generation recombinant $\mathrm{X}$-linked introgressions from $D$. mauritiana in an otherwise pure $D$. simulans genetic background. In parallel, we performed population genomic analyses between $D$. mauritiana and D. simulans to study the chromosomal distributions of interspecific divergence and gene flow. These analyses lead to two discoveries regarding the role of meiotic drive in speciation. First, we find evidence for modest $X$-linked segregation distortion in hybrids, supporting the hypothesis that cryptic sex-ratio systems are common. Second, we show that a now-cryptic X-linked sexratio drive system recently introgressed between species and likely caused large selective sweeps in both species. As a result, this $\mathrm{X}$-linked region shows greatly reduced interspecific sequence divergence and an associated lack of hybrid male sterility factors. Contra the drive hypothesis, in this instance, gene flow at a meiotic drive locus may have prevented or undermined the evolution of $X$-linked hybrid male sterility. These findings suggest that the effects of selfish genetic elements on interspecific divergence and the accumulation of incompatibilities depend on their opportunity to migrate between species during complex speciation.

\section{Results}

\section{Mapping X-linked hybrid male sterility}

Multiple intervals on the $X$ chromosome cause male sterility when introduced from $D$. mauritiana into D. simulans (True et alo, 1996b; Maside et alo, 1998). The number and identities of the causal factors, how they disrupt spermatogenesis, and the evolutionary forces that drove their interspecific divergence are unknown. We therefore generated a high-resolution genetic map of X-linked hybrid male sterility between the two species, with the ultimate aim of identifying a panel of sterility factors. We first introgressed eight $X$-linked $D$. mauritiana segments that together tile across $\sim 85 \%$ of the euchromatic length of the $X$ chromosome into a $D$. simulans genetic background (Figure 1A, $B$; Table 1). Each introgressed segment was marked by two co-dominant $P$ element insertions bearing mini-white transgenes $\left(P\left[w^{+}\right]\right.$; True et alo, 1996a) that serve as visible genetic markers. We introgressed these ' $2 P^{\prime}$ ' segments into the $D$. simulans $w^{\mathrm{XD} 1}$ genetic background through $>40$ generations of repeated backcrossing (Figure 1A). Our ability to generate these introgression genotypes confirms that the distal $85 \%$ of the $D$. mauritiana $X$ euchromatin carries no dominant factors that cause female sterility or lethality in a D. simulans genetic background (True et alo, 1996b; Tao et alo, 2003). All eight $2 P$ introgression genotypes are, however, completely male-sterile, indicating that each of the introgressed regions contains one or more hybrid male sterility factors. Two pairs of introgression genotypes carry largely overlapping introgressed $D$. mauritiana segments and were combined for further analyses $(2 P-5 \mathrm{a} / \mathrm{b}$ and $2 P-6 \mathrm{a} / \mathrm{b}$, respectively; Figure $1 B$, Table 1$)$.

To determine the genetic basis of male sterility within each $2 P$ interval, we generated recombinant introgressions using $D$. simulans strains carrying $p B a c[e Y F P]$ visible markers (Stern et al., 2017) (Figure 1C). These crosses capture unique recombination events between $P\left[w^{+}\right]$and $p B a c[e Y F P]$ markers, allowing recombinant $D$. mauritiana introgressions (hereafter called $1 P-Y F P$ ) to be propagated indefinitely through females without recombination via selection for the 1P-YFP genotype. From these 1P-YFP females, an unlimited number of replicate males carrying identical 1 P-YFP recombinant introgressions can be generated, assayed for male fertility, and archived for genotyping (Figure 1C; see below). We assayed male fertility in at least 10 individual males from each of 617 recombinant 1P-YFP genotypes (Table 2; see Materials and methods), and used the mean number of offspring across replicate males as the measure of fertility for each $1 P$-YFP genotype. Across $1 P$ YFP genotypes, the mean number of offspring ranged from 0 to 215 progeny; 238 genotypes (38.6\%) were completely male-sterile, producing no offspring, and an additional $62(10 \%)$ produced fewer than five offspring per male (Figure 1-figure supplement 1). Of the remaining 1P-YFP genotypes, 231 (37.4\%) had intermediate fertility, and $86(13.9 \%)$ had fertility indistinguishable from pure D. simulans controls $\left(P_{\text {t-test }}>0.01\right)$. 


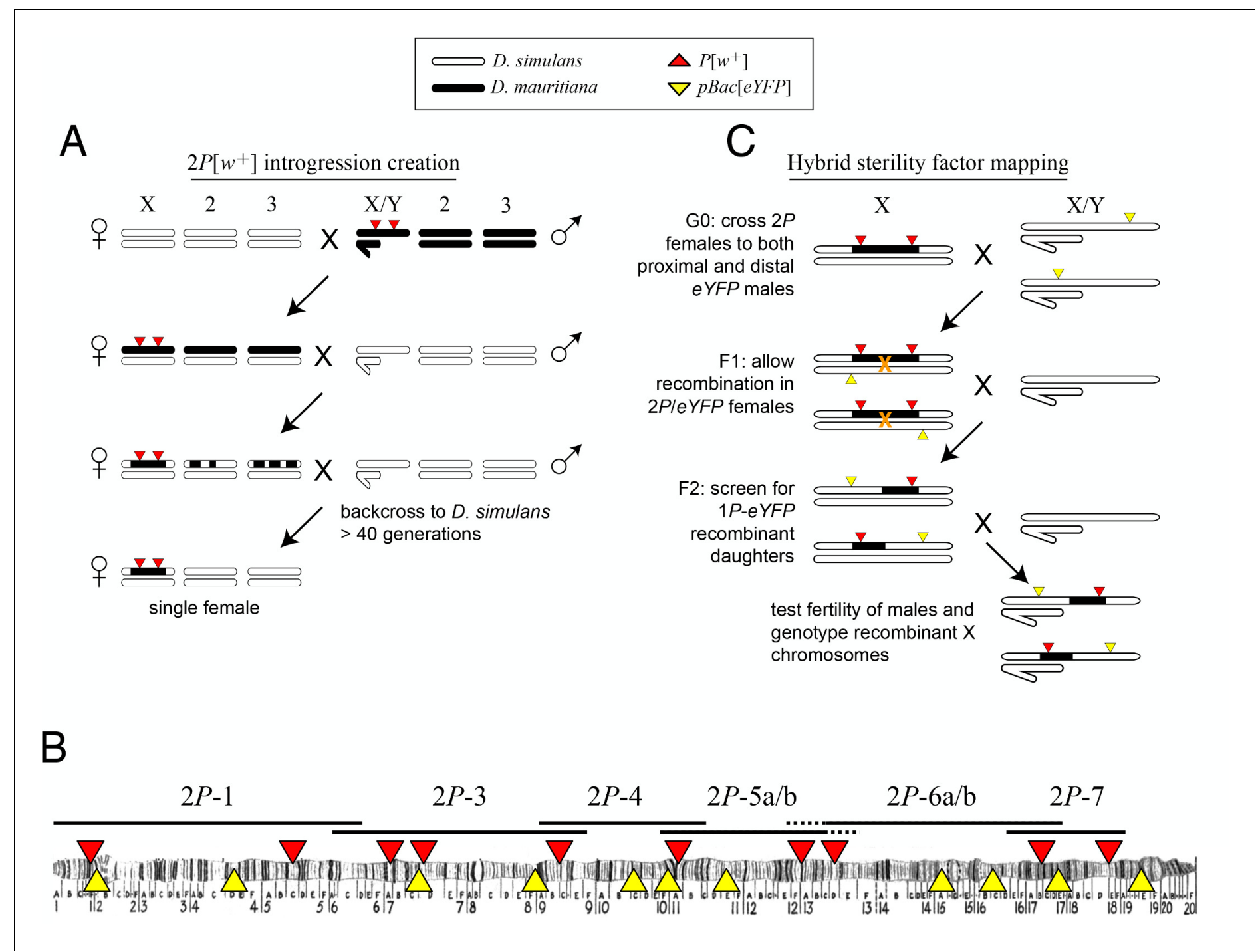

Figure 1. Crosses used to introgress eight regions of the $D$. mauritiana $X$ chromosome into a $D$. simulans genome. (A) $D$. mauritiana ' $2 P^{\prime}$ lines were constructed by combining pairs of $P$-element insertions containing the miniwhite transgene $\left(P\left[w^{+}\right]\right.$; red triangles) distributed across the $X$ chromosome. The $P\left[w^{+}\right]$inserts are semi-dominant visible eye-color markers that permit discrimination of individuals carrying 0,1 or $2 P\left[w^{+}\right]$. $X$-linked segments from $D$. mauritiana were introgressed into a $D$. simulans genetic background by backcrossing $2 P\left[w^{+}\right]$hybrid females to $D$. simulans $w^{X D 1}$ males for over 40 generations. Each introgression line was then bottlenecked through a single female to eliminate segregating variation in the recombination breakpoints flanking the $2 P\left[w^{+}\right]$interval. (B) Cytological map of the $D$. melanogaster $X$ chromosome, indicating the locations of $P\left[w^{+}\right]$and $p B a c[e Y F P]$ transgene insertions. The extent of regions introgressed from $D$. mauritiana into $D$. simulans (e.g. $2 P-1$ ) are labeled above the map. Two pairs of introgression genotypes $(2 P-5 a / b$ and $2 P-6 a / b)$ mostly overlap; the regions included in $2 P-5 b / 2 P-6 b$ but not $2 P-5 a / 2 P-6 a$ are indicated by dashed lines. (C) Meiotic mapping of sterility factors. $2 P\left[\mathrm{w}^{+}\right]$females were crossed to $D$. simulans strains carrying an $X$-linked pBac[eYFP] transgene (yellow triangles) that was used as an additional visible marker to score recombinant chromosomes. Recombinant $X$ chromosomes with both pBac[eYFP] and a single $P\left[w^{+}\right]$were chosen and assayed for male fertility. Recombinant chromosomes were generated using pBac[eYFP] markers both proximal and distal to each $2 P$ introgression.

DOI: https://doi.org/10.7554/eLife.35468.002

The following source data and figure supplement are available for figure 1:

Source data 1. Source data for Figure 1-figure supplement 1, Figure 4-figure supplement 1. DOI: https://doi.org/10.7554/eLife.35468.004

Source data 2. Source data for Figure 1-figure supplement 1, Figure 4-figure supplement 1.

DOI: https://doi.org/10.7554/eLife.35468.005

Source data 3. Source data for Figure 1-figure supplement 1.

DOI: https://doi.org/10.7554/eLife.35468.006

Figure 1 continued on next page 
Figure 1 continued

Figure supplement 1. Distribution of fertility (number of progeny) among all males carrying recombinant $1 P$-YFPX chromosomes, and average number of progeny among all 1 P-YFP genotypes.

We determined high-resolution genotypes of 1 P-YFP recombinant introgressions using multiplexed whole-genome sequencing (Andolfatto et al., 2011). After quality filtering, we obtained high-confidence genome-wide genotype information for 4391 P-YFP recombinant introgressions (Figure 2). No genotype showed evidence for any autosomal D. mauritiana alleles, confirming that the introgression scheme isolated $X$-linked $D$. mauritiana segments in a pure $D$. simulans autosomal genetic background (Figure 2-figure supplement 1). Recombinant $1 P-Y F P$ introgressions on the $X$ chromosome ranged in size from 0.219 to $6.32 \mathrm{Mbp}$, with a mean length of $1.97 \mathrm{Mb}$ (Table 3). Figure 2 shows the distribution of $D$. mauritiana introgression segments and their corresponding sterility phenotypes. Three large regions on the $D$. mauritiana $X$ chromosome can be introgressed into $D$. simulans without strong negative effects on male fertility, indicating an absence of major hybrid male sterility factors in these regions (Figure 2). Conversely, we delineated four small regions $(<700$ $\mathrm{kb}$ ) that consistently and strongly reduced male fertility: $90 \%$ of replicate males with introgressions spanning these regions produce fewer than five offspring. Quantitative trait locus (QTL) analyses confirmed the existence of genetic variation among introgression genotypes that significantly affects male fertility (Figure 3, Figure 3-figure supplement 1). At least five QTL peaks are significant at $\mathrm{p}<0.01$ (permutation test). Most regions containing $D$. mauritiana alleles reduce the average number of progeny to $<15$. Two QTL peaks $(2.5 \mathrm{cM}$, and $29.3 \mathrm{cM}$, Figure 3 ) appear to show higher fertility associated with the $D$. mauritiana allele than the $D$. simulans allele, but this is attributable to $D$. mauritiana sterility factors located at $12.6 \mathrm{cM}$ and $17.5 \mathrm{cM}$ and the negative linkage disequilibrium that is generated across a $2 P$ interval by our meiotic mapping approach (Figure $1 \mathrm{C}$ ).

\section{Sex ratio distortion revealed through experimental introgression}

Among fertile 1 P-YFP males, progeny sex ratios were skewed toward a slight excess of sons: the mean proportion of daughters was 0.45 , and $86 \%$ of fertile 1 P-YFP genotypes (260/303) produced fewer than $50 \%$ daughters (Figure 4). These skewed sex ratios are at least partially attributable to effects of the $\operatorname{sim} w^{\mathrm{XD} 1}$ genetic background, as a similar male bias was observed among progeny of control $\operatorname{sim} w^{\mathrm{XD1}}$ males (mean proportion females $=0.46, n=35$ sires, $t$-test vs. null hypothesis of $0.5, p=0.005)$. We observe a significant positive correlation between fertility and progeny sex-ratio among both sim $W^{\mathrm{XD} 1}$ and introgression genotypes $(\rho=0.44, \mathrm{p}=0.009 ; \rho=0.21, \mathrm{p}=0.0002$, respectively); males that sire fewer progeny sire a lower proportion of daughters (Figure 4-figure supplement 1). However, there is some evidence that introgressed $D$. mauritiana alleles modify this modest male bias: across all fertile introgression genotypes, there is a significant negative correlation between the length of the introgressed $D$. mauritiana segment and the proportion of female progeny produced by that genotype $(\rho=-0.31, \mathrm{p}<0.0001$, Figure 4-figure supplement 2$)$. This

Table 1. Locations and lengths of $2 P$ intervals.

\begin{tabular}{llll}
$2 P$ interval & Left $P\left[w^{+}\right]^{*}$ & Right $P\left[w^{+}\right]^{*}$ & Length (Mbp) \\
\hline $2 P-1$ & 993419 & 4498520 & 3.51 \\
\hline $2 P-3$ & 6192555 & 9126133 & 2.93 \\
\hline $2 P-4$ & 9126133 & 11189873 & 2.06 \\
\hline $2 P-5 a$ & 11189873 & 13324017 & 2.13 \\
\hline $2 P-5 b$ & 11189873 & 13903934 & 2.71 \\
\hline $2 P-6 a$ & 13903934 & 17492084 & 3.59 \\
\hline $2 P-6 b$ & 13324017 & 17492084 & 4.17 \\
\hline $2 P-7$ & 17492084 & 18660037 & 1.17 \\
\hline
\end{tabular}

*coordinate position in the assembled D. simulans $w^{501}$ genome

DOI: https://doi.org/10.7554/eLife.35468.007 
Table 2. Fertility and sex ratio phenotypes for $1 P$-YFP recombinant genotypes.

\begin{tabular}{|c|c|c|c|c|c|c|c|}
\hline $2 P$ interval & $N$ tested & N sterile* & $N$ sub-fertile & $\mathbf{N}$ fertile $^{\dagger}$ & Mean fertility ${ }^{\dagger}$ & $\%$ fertile $^{\dagger}$ & Mean SR ${ }^{\dagger}$ \\
\hline $2 P-1$ & 171 & 48 & 20 & 103 & 72.2 & 0.60 & 0.43 \\
\hline $2 P-3$ & 97 & 12 & 21 & 64 & 67.4 & 0.66 & 0.45 \\
\hline $2 P-4$ & 77 & 17 & 9 & 51 & 71.9 & 0.66 & 0.45 \\
\hline $2 P-5 a / b$ & 92 & 23 & 16 & 53 & 68.2 & 0.58 & 0.51 \\
\hline $2 P-6 a / b$ & 97 & 69 & 10 & 18 & 73.8 & 0.19 & 0.44 \\
\hline $2 P-7$ & 83 & 69 & 6 & 8 & 136.5 & 0.10 & 0.47 \\
\hline all 1P-YFP genotypes & 617 & 238 & 82 & 297 & 81.7 & 0.48 & 0.45 \\
\hline
\end{tabular}

*genotypes where no male produced any offspring

${ }^{\dagger}$ genotypes where at least two males produced at least five offspring

effect seems to be independent of the effects of introgressed alleles on fertility as the partial correlation between progeny sex-ratio and introgression length remains unchanged after taking into account the effect of fertility ( $\rho=-0.31$, $\mathrm{p}<0.0001$; Figure 4 -figure supplement 2$)$. One interpretation of these results is that the $Y$ chromosome of $\operatorname{sim} W^{X D 1}$ causes weak segregation distortion, and the intensity of distortion is modified by $X$-linked alleles at multiple loci from $D$. mauritiana.

Although the majority of fertile $1 P$-YFP genotypes sired male-biased progeny, introgressions that included the distal end of the $2 P-5$ region sired female-biased progeny (Figure 4). QTL analysis of progeny sex ratio confirms a significant peak in the distal portion of $2 P-5$ (Figure 4-figure supplement 3). The estimated effect of this QTL on progeny sex ratios is $54.6 \%$ daughters for the mauritiana allele versus $42.5 \%$ daughters for the simulans allele. These results are consistent with the existence of a cryptic (normally-suppressed) X-linked drive allele in D. mauritiana that is released in a $D$. simulans genetic background, as the $D$. mauritiana $w^{12}$ strain used to generate the $2 P$ introgressions produces slightly male-biased progeny sex-ratios using the same fertility assay (one male paired with three $D$. simulans $w^{X D 1}$ females, $n=10$ sires, mean sex-ratio $=0.47, t$-test vs. $D$. simulans $\left.w^{X D 1} p=0.4\right)$. This region of the $X$ chromosome does not contain any previously mapped meiotic drive loci in D. simulans (Montchamp-Moreau et al., 2006; Tao et al., 2007a; Helleu et al., 2016), suggesting that our experiments have uncovered a novel cryptic drive locus and provide the first evidence of cryptic X-chromosome drive in $D$. mauritiana.

\section{Population genomics of speciation history}

The high density of hybrid male sterility factors and the presence of cryptic drive systems on the $X$ chromosome is expected to influence patterns of gene flow between $D$. mauritiana and $D$. simulans. We therefore analyzed whole-genome variation within and between $10 \mathrm{D}$. mauritiana strains from Mauritius (Garrigan et al., 2014) and 20 D. simulans strains, including nine from Madagascar, ten from Kenya, and one from North America (Rogers et alı, 2014; Hu et al., 2013). These data allow us to characterize differentiation and identify genomic regions with aberrant genealogical histories consistent with recent interspecific introgression. The analyses reported here complement earlier studies that characterized interspecific divergence (Garrigan et al., 2012), polymorphism within D. mauritiana (Garrigan et al., 2014; Nolte et al., 2013), and polymorphism within D. simulans (Begun et al., 2007; Rogers et al., 2014). Below we present genome-wide population genetic analyses using non-overlapping 10-kb windows (unless otherwise stated; see Materials and methods).

\section{Polymorphism}

Our genome-wide analyses provide multiple indicators that the island-endemic $D$. mauritiana has a smaller effective population size than D. simulans (Table 4), consistent with previous multi-locus analyses (Hey and Kliman, 1993; Kliman et al., 2000). Compared to D. simulans, total polymorphism (Nei and Li, 1979) in D. mauritiana is 32\% lower on the $X$ chromosome and $19 \%$ lower on the autosomes (Figure 5-figure supplement 1). The X/autosome ratio of polymorphism is thus lower in $D$. mauritiana (0.656) than in $D$. simulans $(0.778)$ and lower than the $3 / 4$ expected for a random mating population with a 1:1 sex ratio (Garrigan et al., 2014). A substantial fraction of extant 


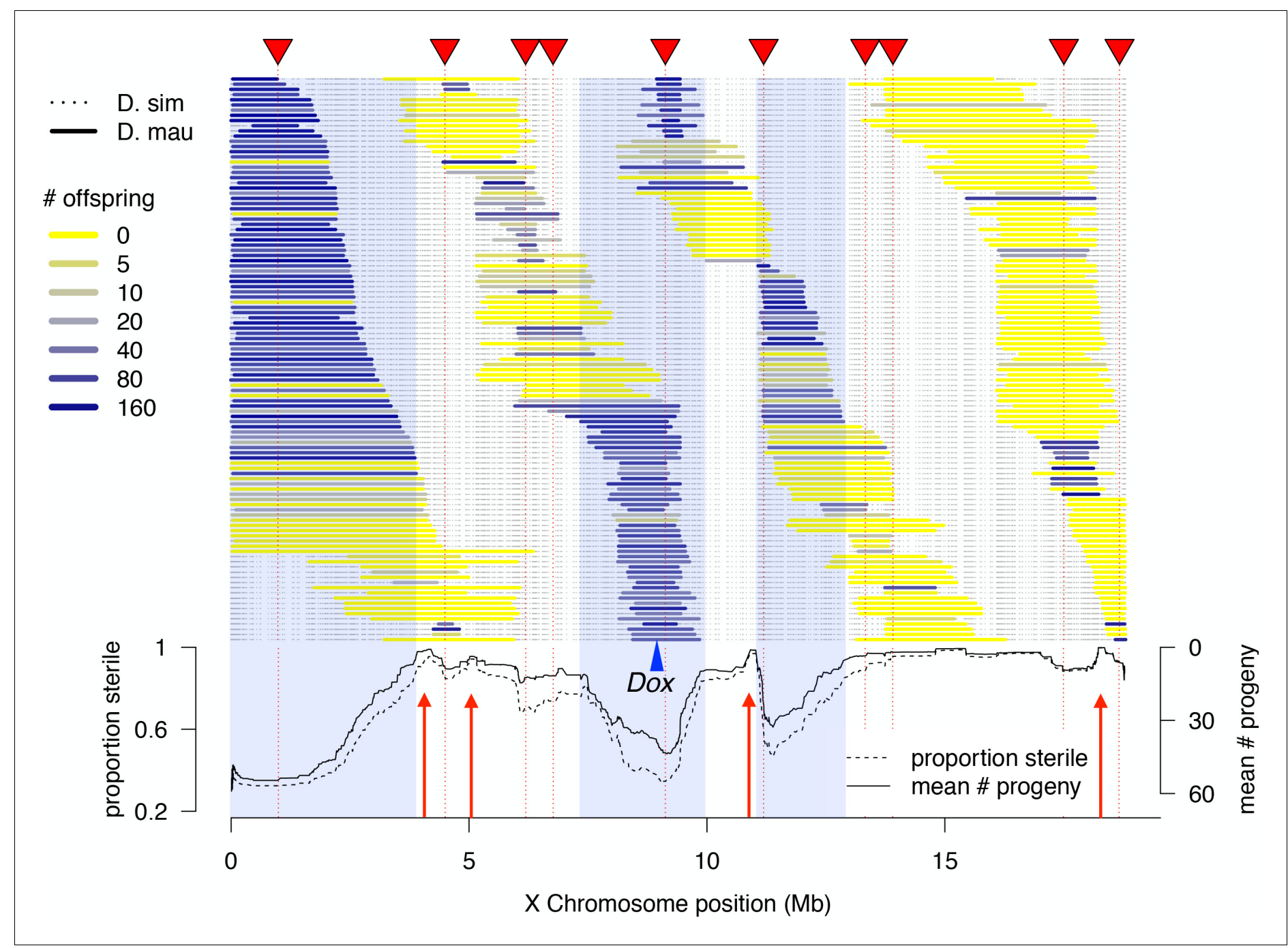

Figure 2. High-resolution genetic map of $X$-linked hybrid male sterility. Colored horizontal bars indicate the extent of introgressed $D$. mauritiana alleles for each recombinant $1 P$-YFPX chromosome. The color of each introgression indicates the mean fertility of 10 replicate males carrying that $1 P$-YFPX chromosome. The three shaded areas indicate fertile regions within which $D$. mauritiana introgressions do not cause sterility, whereas the four red arrows indicate small candidate sterility regions. The blue arrowhead indicates the location of the Dox/MDox meiotic drive loci. Lines in the lower panel indicate the average number of offspring and average proportion of sterile males (defined as producing fewer than five offspring) for all $1 P$-YFP genotypes that carry D. mauritiana alleles at each genotyped SNP.

DOl: https://doi.org/10.7554/eLife.35468.009

The following source data and figure supplement are available for figure 2 :

Source data 1. Source data for Figure 2, Figure 2-figure supplement 1, Figure 4.

DOI: https://doi.org/10.7554/eLife.35468.011

Figure supplement 1. SNP locations and inferred ancestry for five recombinant 1P-YFP genotypes.

DOI: https://doi.org/10.7554/eLife.35468.010

polymorphisms in both species arose in their common ancestor, reflecting the large effective population sizes of both species and relatively recent species split time (see Materials and methods). Compared to $D$. simulans, however, D. mauritiana has retained $74.4 \%$ as many ancestral polymorphisms and accumulated just $46.3 \%$ as many derived polymorphisms. The site frequency spectra (Tajima, 1989) in D. mauritiana are less skewed toward rare variants than in D. simulans, and average linkage disequilibrium (Kelly, 1997) is twofold higher. Overall, these findings show that, relative to D. simulans, D. mauritiana has lower nucleotide diversity; retained fewer ancestral SNPs; accumulated fewer derived SNPs; a less negatively skewed site frequency spectrum; and greater linkage 
Table 3. Distribution of $1 P$-YFP recombinant introgression lengths.

\begin{tabular}{lllll}
$2 P$ interval & Sequenced & Min size & Mean size & Max size \\
\hline $2 P-1$ & 129 & 295,225 & $2,617,833$ & $6,322,871$ \\
\hline $2 P-3$ & 73 & 306,052 & $1,636,944$ & $3,818,569$ \\
\hline $2 P-4$ & 55 & 226,018 & $1,482,659$ & $2,917,578$ \\
\hline $2 P-5$ & 61 & 365,004 & $1,627,632$ & $3,276,930$ \\
\hline $2 P-6$ & 55 & 692,350 & $2,400,499$ & $4,764,204$ \\
\hline $2 P-7$ & 66 & 218,722 & $1,412,108$ & $2,502,552$ \\
\hline
\end{tabular}

DOI: https://doi.org/10.7554/eLife.35468.016

disequilibrium—all patterns consistent with a historically smaller effective population size in D. mauritiana than in D. simulans.

\section{Divergence and differentiation}

Net divergence levels between species are comparable to diversity levels within species. The median number of pairwise differences per site $\left(D_{X Y}\right)$ between the two species, estimated in non-overlapping $10-\mathrm{kb}$ windows, is 0.010 for the $X$ chromosome and 0.013 for the autosomes. However, as the $X$ chromosome has lower levels of polymorphism within species, the median net divergence $\left(D_{A}\right)$ between species is 0.0007 for the $X$ (mean $D_{A}=0.0007$ ) and -0.0005 (mean $D_{A}=-0.0006$ ) for the autosomes (a negative value of $D_{A}$ on the autosomes occurs because, on average, levels of withinspecies polymorphism exceed levels of between-species divergence). $D_{A}$ is significantly greater on the $X$ chromosome than the autosomes $(p<0.0001$ for both medians and means). Allele frequency differentiation is also higher for the $X$ chromosome (median $F_{\mathrm{ST}}=0.378$ ) than the autosomes (median $F_{\mathrm{ST}}=0.279, P_{\mathrm{MWU}}<0.0001$ ). These $F_{\mathrm{st}}$ estimates imply that, for $\mathrm{X}$-linked and autosomal loci, the mean times to coalescence for two gene copies sampled from the different species are 2.2- and 1.8-fold deeper than the mean coalescence times for two gene copies within-species, respectively (Slatkin, 1993).

\section{Recent interspecific gene flow and introgression}

Gene flow between $D$. mauritiana and $D$. simulans has been rare during their speciation history, with an apparent recent increase (Garrigan et al., 2012). To identify genomic regions that have introgressed between species in the recent past, we used the $G_{\min }$ statistic - the ratio of the minimum pairwise sequence distance between species to the average pairwise distance between species ( $\min \left[D_{X Y}\right] / \bar{D}_{X Y} ;$ Geneva et al., 2015). As populations diverge without gene flow, all loci in the genome gradually approach reciprocal monophyly, leaving just one ancestral lineage from each population available for coalescence in the ancestral population. Consequently, the minimum distance (numerator) equals the mean pairwise distance (denominator), causing $G_{\min } \rightarrow 1$ with zero variance. Conversely, $G_{\min }$ is small when the minimum distance is small relative to the mean pairwise distance. $G_{\min }$ is therefore sensitive to genealogical configurations resulting from recent gene flow, particularly when introgressed haplotypes segregate at low to intermediate population frequency in at least one of the populations (Geneva et al., 2015). Importantly, $G_{\min }$ distinguishes genealogies produced by introgression from those produced by incomplete lineage sorting. Between $D$. mauritiana and $D$. simulans, we find that median $G_{\min }$ ( \pm median absolute deviation) estimated for $10-\mathrm{kb}$ windows across the major chromosome arms ranges from $0.761 \pm 0.0537$ for $3 L$ to $0.785 \pm 0.0531$ for the $X$ (Figure 5 ; Kruskal-Wallis test, $p<0.0001)$. As $95 \%$ of $G_{\min }$ values are $<0.85$, reciprocal monophyly for $10-k b$ windows is rare.

To identify $10-\mathrm{kb}$ outlier windows that have genealogical histories inconsistent with strict allopatric divergence, we used a Monte Carlo simulation procedure that assumes a constant species divergence time across all 10-kb intervals, separately for the $X$ and the autosomes (see Materials and methods). In total, 196 of the 10,443 10-kb windows (1.9\%) have a more recent common ancestry between $D$. mauritiana and $D$. simulans than expected under a strict allopatric divergence model, as indicated by significantly low values of $G_{\min }(P \leq 0.001$, corresponding to a genome-wide false discovery rate of $5 \%$ ). As $G_{\min }$ is a ratio, significantly small $G_{\min }$ values could result from unusually small 


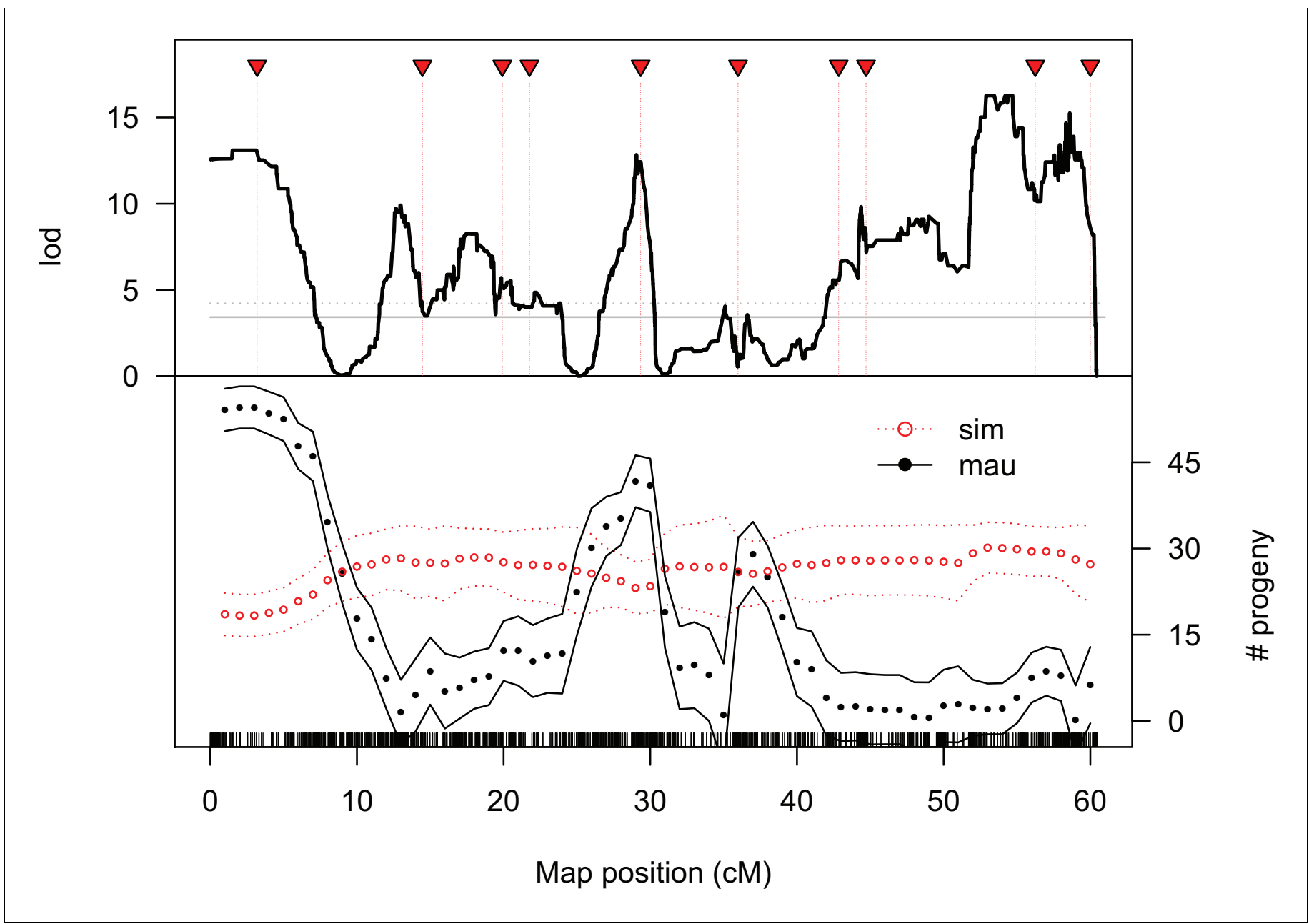

Figure 3. QTL analysis of male fertility. Mean offspring counts for each genotype were transformed as $\log _{10}(N+1)$. The top plot shows lod scores for a two-part model that treats completely sterile genotypes as one class, and tests for quantitative effects on fertility among non-sterile genotypes. The solid and dotted gray lines indicate $5 \%$ and $1 \%$ significance thresholds, respectively, determined from 10,000 permutations. The bottom plot shows the estimated effects of D. simulans and D. mauritiana alleles at QTL placed every $1 \mathrm{cM}$ (bounding lines indicate $95 \%$ confidence intervals).

DOI: https://doi.org/10.7554/eLife.35468.012

The following source data and figure supplements are available for figure 3 :

Source data 1. Source data for Figure 3, Figure 3-figure supplement 1, Figure 3-figure supplement 2, Figure 4-figure supplement 3. DOI: https://doi.org/10.7554/eLife.35468.015

Figure supplement 1. Alternate QTL models of male fertility.

DOl: https://doi.org/10.7554/eLife.35468.013

Figure supplement 2. QTL analysis of male fertility incorporating introgression length as a covariate.

numerators (minimum $\left.D_{X Y}\right)$ or unusually large denominators $\left(\bar{D}_{X Y}\right)$. We find that 10-kb windows with significant $G_{\min }$ values have smaller median minimum $D_{X Y}$ (0.0056 in introgression windows versus 0.0094 genome-wide, $\left.P_{M W U}<0.0001\right)$ as well as smaller median $\bar{D}_{X Y}(0.0110$ in introgression windows versus 0.0124 genome-wide $P_{\mathrm{MWU}}<0.0001$ ), indicating that the significant $G_{\min }$ values are due to unusually small minimum $D_{X Y}$ values. The smaller $\bar{D}_{X Y}$ of windows with significant $G_{\min }$ reflects the contribution of the introgressed, low-distance haplotypes to the overall average pairwise distance between species.

Introgression windows are 4.4-fold underrepresented on the $\mathrm{X}$ chromosome: only nine of 1842 $10-\mathrm{kb}$ windows on the $\mathrm{X}$ chromosome (0.49\%) have significant $G_{\min }$ values versus 187 of 8601 10-kb windows on the autosomes (2.17\%; Fisher's exact test $\mathrm{p}<0.0001)$. However, not all 10-kb 


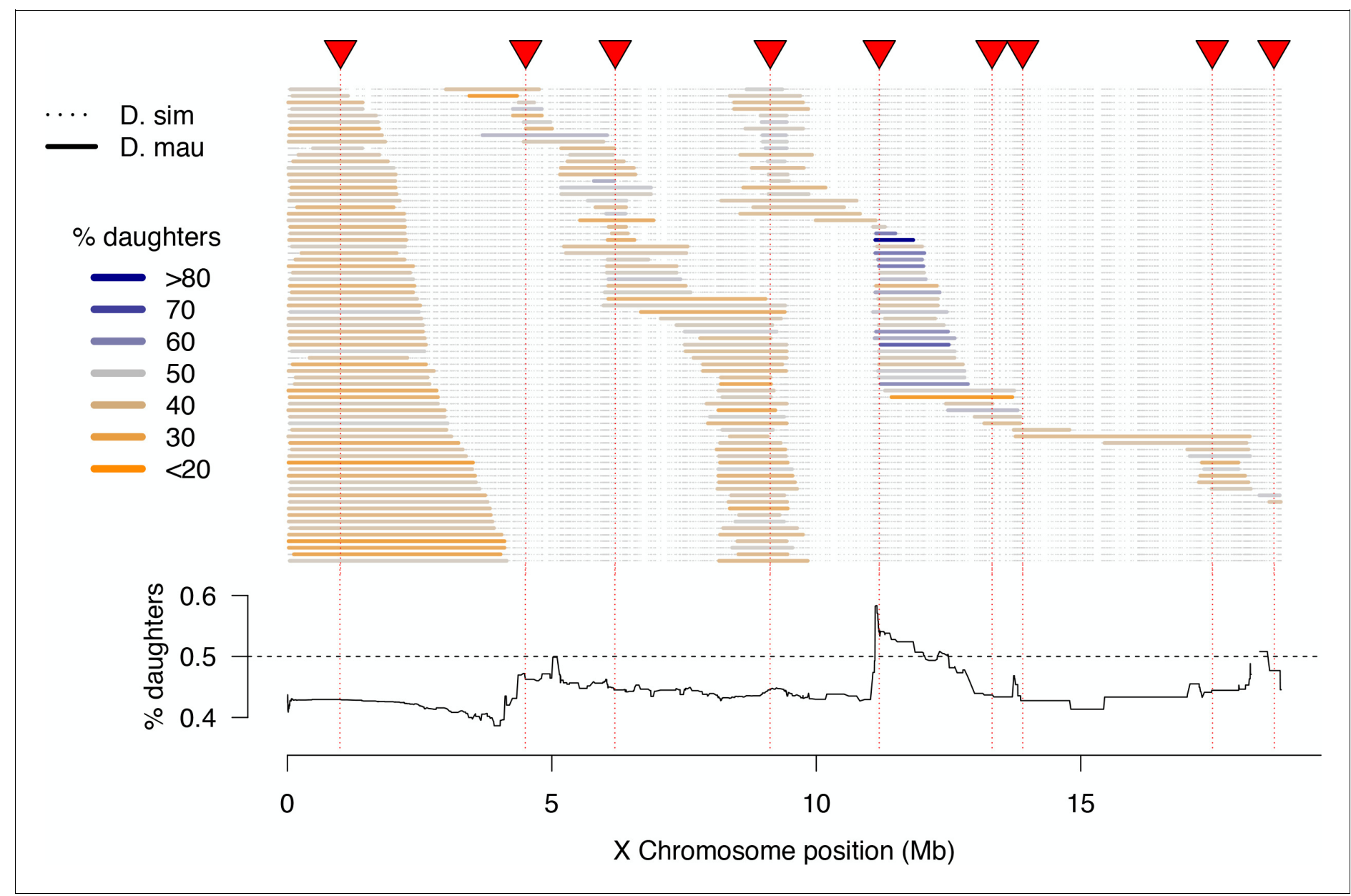

Figure 4. High-resolution map of progeny sex ratios among fertile 1 -YFP introgression male genotypes. Colored horizontal bars indicate the extent of introgressed $D$. mauritiana alleles for each fertile recombinant $1 P$-YFP $X$ chromosome. The color of each introgression indicates the sex-ratio of progeny from replicate males carrying that $1 P$-YFP $X$ chromosome. The line below indicates the average progeny sex-ratio for all $1 P$-YFP genotypes that carry $D$. mauritiana alleles at each genotyped SNP.

DOI: https://doi.org/10.7554/eLife.35468.017

The following figure supplements are available for figure 4:

Figure supplement 1. Relationship between progeny number and sex-ratio.

DOI: https://doi.org/10.7554/eLife.35468.018

Figure supplement 2. Relationship between introgression length, fertility, and sex-ratio.

DOI: https://doi.org/10.7554/eLife.35468.019

Figure supplement 3. QTL analysis of progeny sex ratio associated with introgression genotypes.

DOI: https://doi.org/10.7554/eLife.35468.020

introgression windows are independent: 169 of the 196 significant 10-kb windows (86.2\%) can be arrayed into contiguous (or nearly contiguous) genomic regions (see Materials and methods). As a result, we infer 27 small (10-kb) introgressions and 21 larger introgressions ranging in size from 20 $\mathrm{kb}$ to $280 \mathrm{~kb}$ (Supplementary file 1). Of these 48 total introgressions, only one is on the $\mathrm{X}$ chromosome and 47 are on autosomes $\left(\chi^{2}\right.$-test, $\left.p=0.0124\right)$. The lengths of these introgressed haplotypes depend on their time spent in the receiving population and on the local recombination rate. First, recombination has eroded introgression sizes over time, with longer, presumably younger, introgressions having smaller average $G_{\min }$ values (Spearman $\rho=-0.6293, p<0.0001$ ) and smaller minimum $D_{\text {xy }}$ values $(\rho=-0.3677, \mathrm{p}=0.0101)$. Second, local recombination rate has been an important factor in determining introgression lengths, with relatively long introgressions tending to reside in chromosomal environments with low rates of crossing over $(\rho=-0.366, \mathrm{p}=0.0105)$. 
Table 4. Population genomics summary statistics.

\begin{tabular}{|c|c|c|c|c|}
\hline Inference & Statistic* & D. simulans & D. mauritiana & $P$-value \\
\hline \multirow[t]{5}{*}{ Polymorphism } & median $\pi_{X}$ & 0.0119 & 0.0076 & $<0.0001^{\ddagger}$ \\
\hline & median $\pi_{\mathrm{A}}$ & 0.0152 & 0.0116 & $<0.0001^{\ddagger}$ \\
\hline & SNPs with inferred ancestry ${ }^{\dagger}$ & $4,324,740$ & $2,181,959$ & $<0.0001^{\S}$ \\
\hline & $\%$ ancestral SNPs & 14.6 & 21.6 & \multirow[t]{2}{*}{$<0.0001^{\#}$} \\
\hline & $\%$ derived SNPs & 85.3 & 78.3 & \\
\hline \multirow[t]{2}{*}{ Site frequency spectra } & median Tajima's $D_{x}$ & -1.218 & -0.536 & $<0.0001^{c}$ \\
\hline & median Tajima's $D_{A}$ & -1.127 & -0.359 & $<0.0001^{c}$ \\
\hline \multirow[t]{2}{*}{ Linkage disequilibrium } & median $Z_{\mathrm{ns}, \mathrm{x}}$ & 0.056 & 0.122 & $<0.0001^{c}$ \\
\hline & median $Z_{\mathrm{ns}}$ A & 0.058 & 0.129 & $<0.0001^{c}$ \\
\hline
\end{tabular}

*Summary statistics estimated from 10-kb non-overlapping windows.

†SNP were inferred as ancestral or derived using parsimony, with D. melanogaster as an outgroup (see Materials and methods).

${ }^{\ddagger} P$-value for Mann-Whitney U-test.

$\S$-value for $\chi^{2}$-test.

\#P-value from Fisher's exact test.

DOI: https://doi.org/10.7554/eLife.35468.021

To complement our distance-based $G_{\min }$ analyses, we also used a genealogy-based four-population (ABBA-BABA) test, summarized by Patterson's D-statistic (Green et al., 2010; Durand et al., 2011), to evaluate the distribution of shared derived variants between $D$. mauritiana and $D$. simulans. Assuming a (( $D$. sechellia, D. simulans), D. mauritiana), D. melanogaster) tree topology, the null expectation is that a history involving zero gene flow should result in approximately equal numbers of $A B B A$ and BABA nucleotide site configurations via lineage sorting, where $A$ and $B$ correspond to ancestral and derived states, respectively (Green et al., 2010; Durand et al., 2011). Instead, we find that $D=0.0812$ (s.e. $=0.0033$; block jackknife with $1 \mathrm{Mb}$ blocks) across the genome, indicating a significant excess of shared derived sites between $D$. simulans and $D$. mauritiana compared to $D$. sechellia and $D$. mauritiana. These findings provide complementary support for a history of interspecific gene flow between $D$. mauritiana and D. simulans.

Interspecific introgression of the cryptic Winters sex-ratio drive system The single introgression detected on the $X$ chromosome corresponds to a 130 -kb region that comprises eight protein-coding genes plus the Winters sex-ratio meiotic drive genes, Distorter on the $X$ (Dox) and, its progenitor gene, Mother of Dox (MDox) (Tao et al., 2007a) (Figure 6). The median $G_{\min }$ value across this $130-\mathrm{kb}$ region is 0.333 , a $\sim 2.4$-fold reduction relative to background $G_{\min }$ on the $\mathrm{X}$ chromosome $\left(P_{\mathrm{MWU}}<0.0001\right)$. The most extreme $10-\mathrm{kb}$ window within the $130-\mathrm{kb}$ region has a minimum $D_{X Y}$ value $(=0.00087)$ that is $92 \%$ smaller than the $X$ chromosome-wide $\bar{D}_{X Y}$, implying that introgression occurred in the recent past. The $130-\mathrm{kb}$ region is also an outlier with respect to Patterson's $D$ statistic: we observe 90.2 (72\%) ABBA sites versus just 35.2 (28\%) BABA sites in the region $(D=0.4382)$, whereas a significantly different configuration of ABBA and BABA sites occurs on the $X$ chromosome outside the 130-kb region (9774.6 [55\%] and 7911.1 [45\%], respectively; $D=0.1054$; $\chi^{2}$-test, $\left.p=0.00027\right)$. The elevated value of $D$ within the $130-\mathrm{kb}$ region indicates a significant excess of derived nucleotide variants shared between $D$. simulans and $D$. mauritiana compared to genomic background levels. Given the evidence from both distance- and genealogy-based analyses, we conclude that this 130-kb haplotype has a history of recent gene flow between species. In D. simulans, when unsuppressed, MDox and Dox cause biased transmission of the $\mathrm{X}$ chromosome during spermatogenesis, with male carriers siring more than $80 \%$ daughters (Tao et al., 2007a). These drivers are suppressed by an autosomal gene, Not much yin (Nmy), a retrotransposed copy of Dox that is a source of endogenous siRNAs that silence both MDox and Dox (Tao et al., 2007b). In non-African D. simulans populations, Dox, MDox, and Nmy are nearly fixed, although haplotypes lacking functional copies of the genes segregate at low frequencies (Kingan et al., 2010). All three loci have histories consistent with selective sweeps in multiple populations of $D$. simulans due to the presumed 


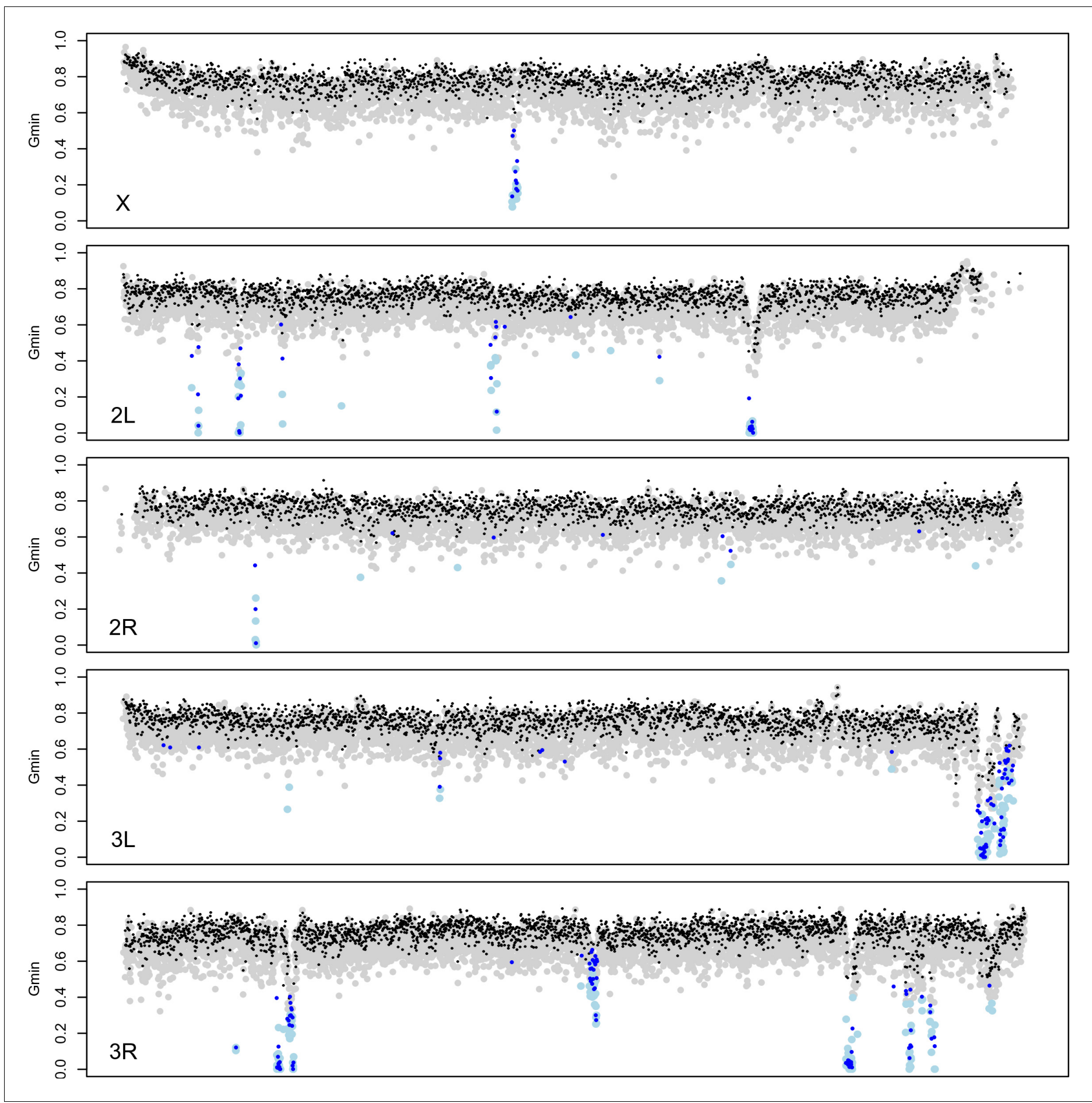

Figure 5. Identification of introgessed regions by $G_{\min }$. Grey (black) dots indicate $G_{\min }$ values calculated using 5-kb (10-kb) windows; light blue (dark blue) dots indicate $5-\mathrm{kb}(10-\mathrm{kb})$ windows with significant $G_{\min }$ values. As with 10-kb windows, 5-kb windows with significant $G_{\min }$ values are 4 -fold underrepresented on the $X$ chromosome: 14 of 36035 -kb windows on the $X$ chromosome $(0.39 \%)$ have significant $G_{\text {min }}$ values versus 266 of 17,0655 -kb windows on the autosomes (1.56\%; Fisher's exact test $p<0.0001)$.

DOI: https://doi.org/10.7554/eLife.35468.022

The following source data and figure supplements are available for figure 5:

Source data 1. Source data for Figure 5-figure supplements 1 and 2.

DOI: https://doi.org/10.7554/eLife.35468.025

Source data 2. Source data for Figure 5.

Figure 5 continued on next page 
Figure 5 continued

DOI: https://doi.org/10.7554/eLife.35468.026

Figure supplement 1. Population genomic scans for polymorphism, divergence, and introgression in 10-kb windows.

DOI: https://doi.org/10.7554/eLife.35468.023

Figure supplement 2. Polymorphism and $G_{\min }$.

DOI: https://doi.org/10.7554/eLife.35468.024

transmission advantage at MDox and Dox and the associated selective advantages of suppressing drive and restoring equal sex ratios at $N$ my (Kingan et al., 2010). We estimated the probability that a random X-linked 130-kb introgression might include Dox and MDox by chance by permuting the location of a 130-kb segment on the $X$ chromosome. Out of 100,000 such random permutations, 356 included Dox and MDox $(p=0.004)$. We hypothesize that the signature of recent introgression at these sex-ratio distorters is not coincidental, but rather that introgression was mediated by their biased transmission through males.

Maximum-likelihood phylogenetic trees for the 130-kb MDox-Dox region show reduced diversity within $D$. mauritiana and reduced divergence between the two species (Figure 6). Among the $10 D$. mauritiana sequences, nucleotide diversity is just $24 \%(\pi=0.0018)$ of background diversity levels on the $\mathrm{X}$ chromosome, corresponding to a massive selective sweep in the $D$. mauritiana genome $\left(P_{\mathrm{MWu}}<0.0001\right.$; see also (Nolte et al., 2013; Garrigan et al., 2014)). The distribution of variability among haplotypes in the $D$. simulans samples is consistent with a parallel, albeit incomplete, selective sweep (Figure 6).

To determine if the MDox and/or Dox drive elements are associated with introgression between species and the selective sweeps within each species, we determined MDox and Dox presence/ absence status for each line using diagnostic restriction digests (see Materials and methods). In contrast to previous work showing that MDox and Dox are nearly fixed among $D$. simulans samples collected outside of Africa (Kingan et al., 2010), we find that the drivers are at lower frequency among our 19 African samples (9 Madagascar, 10 Kenya): five have MDox (26\%), five have Dox (26\%), and only one has both genes (5\%; NS33; Supplementary file 2). Despite these low frequencies, MDox and Dox are overrepresented among the haplotypes shared between species: 6 of the 7 shared haplotypes have MDox and/or Dox (Fisher's Exact $P_{\mathrm{FET}}=0.0018$ ), and 2 of the 7 possess both drivers $\left(P_{\mathrm{FET}}=0.0158 ; n=19\right.$ African samples, plus the reference strain, $D$. simulans $w^{501}$, which has both). In D. mauritiana, all 10 lines have MDox, but only two have Dox (Figure 6; Supplementary file 2). RT-PCR shows that MDox is expressed in testes from both species (see Materials and methods), confirming its potential activity. These findings provide support for the hypothesis that segregation distortion mediated by Dox and (transcriptionally active) MDox genes was responsible for introgression and the parallel sweeps at this locus.

Notably, the large MDox-Dox introgression, and its associated sweep co-localize with one of the three regions of the $X$ chromosome that, in our mapping experiments, fails to cause male sterility when introgressed from $D$. mauritiana into $D$. simulans (Figure 2). These observations suggest that a driving haplotype moved between species and swept to high frequency in $D$. simulans and fixation in $D$. mauritiana, thereby reducing local sequence divergence between species. This discovery has two implications. First, the MDox-Dox region is the only locus on the $\mathrm{X}$ chromosome to have recently escaped from its linked hybrid incompatibility factors and introgressed between species. Second, by sweeping to high frequency or fixation, the MDox-Dox drive element region reduced local divergence between species and, incidentally, undermined the accumulation of genetic incompatibilities that might cause hybrid male sterility.

\section{Discussion}

Our combined genetic and population genomics analysis of hybrid male sterility and gene flow between $D$. mauritiana and $D$. simulans yields three findings. First, we confirm the rapid accumulation of X-linked hybrid male sterility between these species and map four major sterility factors to small $(<700 \mathrm{~kb}$ ) intervals (Figure 2). Second, we find that very recent natural introgression has occurred between these species, albeit almost exclusively on the autosomes, consistent with a large $X$-effect on gene flow (Supplementary file 1). Third, we discover new roles for meiotic drive during 


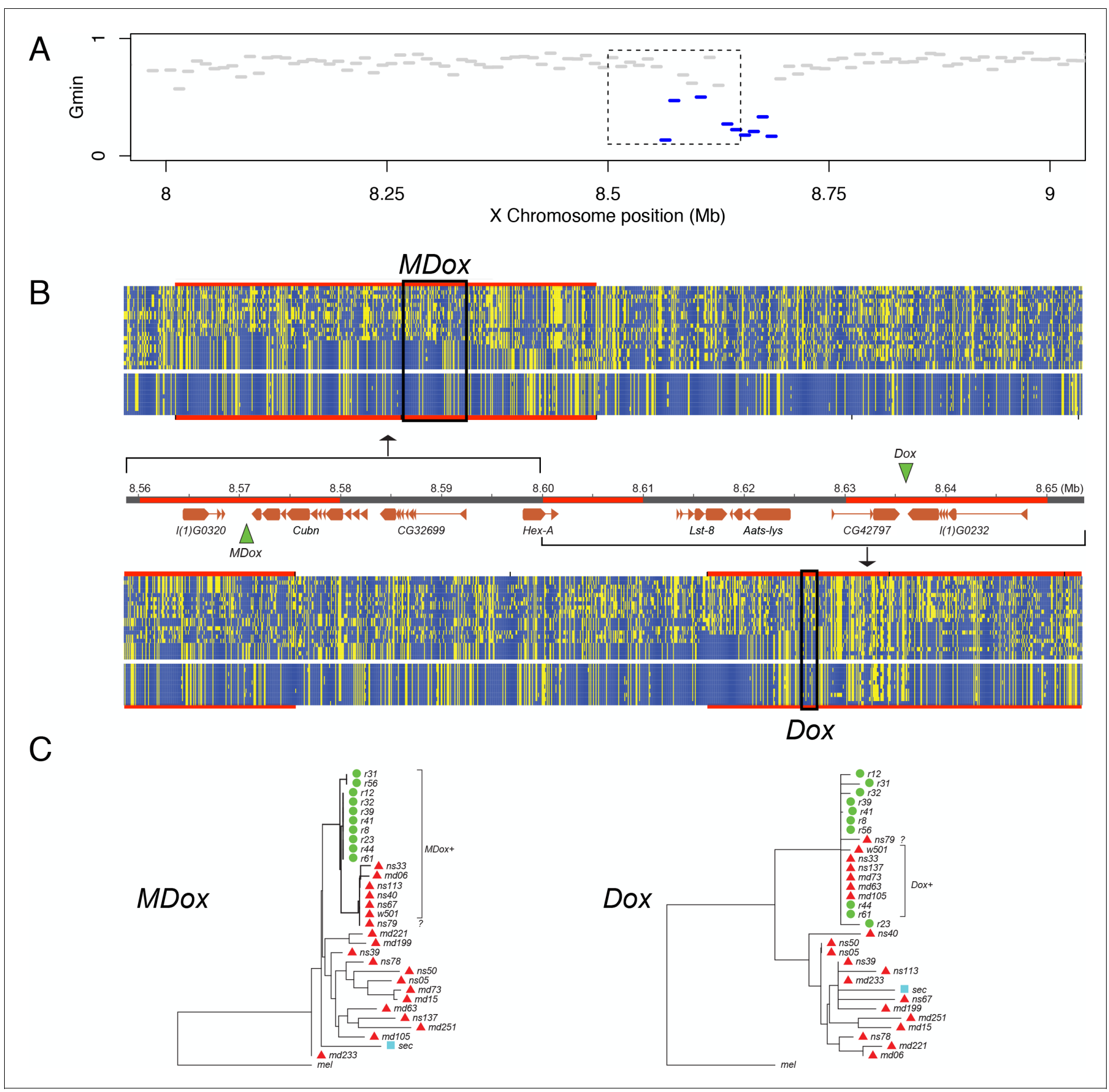

Figure 6. Natural introgression of the MDox-Dox region of the $X$ chromosome. (A) $G_{\min }$ values for $10-k b$ windows in the region containing $M D$ ox and Dox. Blue lines indicate windows with significantly low $G_{\min }$ values. Inset box indicates the 90-kb region shown in panel B. (B) DNA polymorphism tables: the top table corresponds to the MDox region, and the bottom corresponds to the Dox region. Within the tables, yellow squares denote the derived nucleotide state, and blue squares indicate the ancestral state. The top 20 rows of each table correspond to the $D$. simulans samples, and the bottom 10 rows correspond to the $D$. mauritiana samples. The genome map between the polymorphism tables shows gene models for the region (orange boxes) and the locations of the MDox and Dox genes (green triangles). Regions highlighted in red are 10-kb windows with significantly low $G_{\min }$ values. (C) Maximum likelihood phylogenetic trees for the MDox and Dox regions. Green circles and red triangles denote D. mauritiana and D. simulans samples, respectively.

DOI: https://doi.org/10.7554/eLife.35468.027

The following source data is available for figure 6:

Figure 6 continued on next page 
Figure 6 continued

Source data 1. Source data for Figure 6.

the history of speciation between these species. Some drive seems to be associated with functional divergence between species: one region of the $D$. mauritiana $X$ chromosome appears to cause segregation distortion in a $D$. simulans genetic background. In contrast, the well-characterized $X$-linked Winters sex ratio distorters, MDox and Dox, have clearly migrated between species, reducing local interspecific divergence. Together, these findings, respectively, suggest that genetic conflict may both promote as well as undermine the special role of sex chromosomes in speciation.

\section{Genetic basis of X-linked hybrid male sterility}

Our genetic analyses were initiated by introgression of six different regions of the $D$. mauritiana $X$ chromosome into a pure D. simulans genetic background. All six regions cause complete hybrid male sterility and therefore carry at least one, or a combination of, D. mauritiana allele(s) that disrupt spermatogenesis due to incompatibilities with X-linked, Y-linked, or autosomal $D$. simulans alleles. Only three large $(>2 \mathrm{Mb}$ ) regions of the $D$. mauritiana $X$ are readily exchangeable between species, permitting male fertility in a $D$. simulans genome. Thus, after only $\sim 250,000$ years, sufficient $X$-linked hybrid male sterility has accumulated to render most of the $D$. mauritiana $X$ chromosome male-sterile on a $D$. simulans genetic background (True et al., 1996b). Most of the $D$. mauritiana $X$ chromosome is male-sterile in a D. sechellia genome as well (Masly and Presgraves, 2007). The combination of such extensive reproductive isolation with such modest genetic divergence makes this species group an ideal system to study the genetic basis of speciation.

We were able to define four small regions $(<700 \mathrm{~kb}$ ), each sufficient to cause complete male sterility (Figure 2), suggesting that these may contain single, strong sterility factors. We also find a large region spanning most of $2 P-6$ from which we were unable to recover fertile $1 P-Y F P$ recombinants. We infer that $2 P-6$ contains a minimum of two strong sterility regions, one tightly linked to each of the flanking $P$-elements (Figure 3 ). While our $2 P$ mapping scheme is designed to facilitate the identification of male sterility factors, the $2 P-6$ interval highlights one of its limitations: in regions like $2 P-6$, for which strong sterility factors are very close to both flanking $P$-elements, we cannot determine how many additional sterility factors might localize to the middle of the interval. The present experiments therefore provide only a minimum estimate of the total number of hybrid male sterility factors on the $X$ chromosome. We tentatively conclude that, within the fraction of the $D$. mauritiana $X$ chromosome investigated, there are at least six genetically separable regions, each individually sufficient to cause virtually complete male sterility. It is worth noting that these experimental approaches detect relatively large-effect sterility factors under a single set of laboratory conditions. There are likely many hybrid male sterility factors of smaller effect, generally neglected in the lab but easily detected by selection in natural populations and thus able to affect the probability of migration at linked loci.

\section{Genomic signatures of complex speciation with gene flow}

The two species studied here are allopatric: $D$. simulans has never been reported on Mauritius, and D. mauritiana has never been found anywhere other than Mauritius (David et al., 1989; Legrand et al., 2011). D. mauritiana appears to have originated from a D. simulans-like ancestor, probably from Madagascar, that migrated and established a population on Mauritius (Hey and Kliman, 1993; Kliman et al., 2000). Our characterization of genome-wide variation within and between $D$. mauritiana and $D$. simulans confirms a coalescent history that reaches considerably deeper into the past than the inferred species split time of $\sim 250,000$ years (Hey and Kliman, 1993; Kliman et al., 2000). Nested within this largely shared coalescent history, many functional differences have evolved between the two species, including extreme ones that mediate large-effect hybrid incompatibilities. The signatures of gene flow found in the genomes of these species imply recurrent bouts of migration and interbreeding. To introgress between species, immigrating foreign haplotypes must escape their locally disfavored chromosomal backgrounds by recombination before being eliminated by selection against linked incompatibilities and locally maladaptive alleles 
(Petry, 1983; Bengtsson, 1985; Barton and Bengtsson, 1986). Conditional on escape, the lengths of foreign haplotypes will be subject to gradual erosion by recombination with the resident genetic background.

Here, and in previous work (Garrigan et al., 2012), we detect evidence consistent with weak migration: $2-5 \%$ of the genome shows evidence of introgression between $D$. simulans and $D$. mauritiana during their recent history. Our population genomic analysis identified 48 segregating foreign haplotypes. We find evidence that the genomic locations and lengths of introgressed foreign haplotypes have been shaped by selection and by recombination in the receiving population. First, selection has likely affected the genomic distribution of foreign haplotypes: only one of the 48 introgressions occurs on the $\mathrm{X}$ chromosome. The opportunity for foreign haplotypes on the $\mathrm{X}$ chromosome to escape linked incompatibilities via recombination is more constrained than on the autosomes, as the $X$ has a higher density of incompatible alleles, and hemizygous selection eliminates foreign X-linked haplotypes more quickly (Muirhead and Presgraves, 2016). Second, we find that the lengths of introgressed haplotypes depend on local recombination rates: introgressions tend to be longer in chromosomal regions with relatively lower recombination rates. Third, after escaping locally deleterious chromosomal backgrounds, recombination eroded the lengths of foreign haplotypes over time: recently introgressed, and hence less diverged, haplotypes tend to be longer. It is worth noting here that the $10-\mathrm{kb}$ windows used for our $G_{\min }$ scan for foreign haplotypes almost certainly fails to identify very small and/or old introgressions. However, similar results are obtained from $G_{\min }$ scans using $10-\mathrm{kb}$ and $5 \mathrm{~kb}$ windows (Figure 5).

\section{Meiotic drive and complex speciation}

The original drive theory posits that hybrid incompatibilities accumulate as incidental by-products of recurrent bouts of meiotic drive and suppression (Hurst and Pomiankowski, 1991; Frank, 1991). Our mapping experiments provide no direct evidence in support of this theory in $D$. mauritiana and D. simulans, as no hybrid male sterility loci co-localized with sex-ratio loci. Direct genetic evidence that sex-ratio distortion is responsible for the evolution of hybrid male sterility is however inherently difficult to obtain, as sterile males produce no offspring, preventing detection of biased sex-ratios. Indeed, the dual role of Ovd in hybrid male sterility and sex-ratio distortion in D. pseudoobscura was only detectable because males recover low levels of fertility as they age (Orr and Irving, 2005). Although weakly fertile males (producing fewer than five offspring) were removed from the sex-ratio analyses presented here, these males show no evidence for systematically biased sex ratios (Figure 4-figure supplement 1).

Our genetic mapping experiments have, however, provided new evidence for the accumulation of cryptic sex-ratio drive systems. We mapped a small region of the $D$. mauritiana $X$ that, when introgressed into a naive $D$. simulans genetic background, causes modest segregation distortion resulting in female-biased progeny sex ratios (Figure 4). As the D. mauritiana X-drive locus does not map to the location of any of the three cryptic drivers known from $D$. simulans, we infer that it may be a new, previously undiscovered drive system in $D$. mauritiana.

Across $D$. simulans and D. mauritiana, four cryptic drive systems have been identified so far: two $\mathrm{X}$-drive systems in D. simulans (Paris and Durham); one X-drive system in D. mauritiana (see above); and one X-drive system found in both species (Winters; see below). We regard this as a minimum for several reasons. First, weak segregation distortion that may be powerful in natural populations can go undetected in laboratory experiments. Second, cryptic drive systems may not be fixed within species, and our genetic mapping experiments have only surveyed genotypes derived from one strain each of $D$. mauritiana and $D$. simulans. Third, no study has yet comprehensively assayed $D$. simulans material introgressed into a D. mauritiana genetic background. Finally, some cryptic drive alleles might go to fixation and then simply degenerate because, once fixed (or suppressed), a driver is in a race: either suffer mutational decay or acquire a mutation that confers a new bout of drive. These considerations-and the discovery of multiple alternative cryptic drive systems in closely related species-imply that sex chromosome drive is not infrequent during the history of species divergence (Jaenike, 2001).

We have found that the Winters sex-ratio drivers, MDox and Dox, have migrated between these two species. The two drivers are suppressed by the autosomal suppressor, Nmy, which is present in both D. simulans and D. mauritiana (Tao et al., 2007a). The general absence of drive in wild-type genotypes of either species raises one of two possibilities. Either Nmy has evolved quickly to 
suppress the newly introgressed MDox and Dox alleles or, alternatively, a suppressing allele of Nmy also introgressed between species. We are unable to distinguish these possibilities with the present data, as Nmy resides in a chromosomal region dense with complex repetitive sequences that are refractory to genome assembly using short-read data.

The discovery that the MDox and Dox drivers have moved between species highlights an implicit assumption of the drive theory of the large X-effect-namely, that species evolve in strict allopatry. With gene flow, drive elements (and other selfish genes) have the opportunity to jump species boundaries and undermine divergence in a process analogous to adaptive introgression (Seehausen et al., 2014; Crespi and Nosil, 2013). The t-haplotype has, for instance, introgressed between sub-species of house mouse, Mus musculus (Macaya-Sanz et alı, 2011). Between D. mauritiana and $D$. simulans, the $G_{\min }$ statistic and the genealogies associated with the MDox-Dox introgressed haplotype (Figure 6) are agnostic on the direction of introgression. Nonetheless, the finding that a drive element crossed a species boundary has important implications for the drive theory explanation of Haldane's rule and the large X-effect. For MDox and Dox to introgress between species, three things must be true: (1) neither MDox nor Dox alleles from the donor species caused male sterility in the recipient species; (2) no X-linked hybrid male sterility factors were so tightly linked to MDox and Dox as to prevent their eventual escape by recombination into the recipient species genetic background; and (3) any sterility factors located within the introgressed region of the recipient $X$ will have been replaced by foreign alleles. Together, these inferences suggest that $a$ selfish drive system was able to invade a new species by not causing male sterility and, for one X-linked region, may have impeded or undone the evolution of hybrid male sterility.

\section{Materials and methods}

Key resources table

\begin{tabular}{|c|c|c|c|c|}
\hline $\begin{array}{l}\text { Reagent type } \\
\text { (species) or resource }\end{array}$ & Designation & Source or reference & Identifiers & Additional information \\
\hline $\begin{array}{l}\text { Genetic reagent } \\
\text { (Drosophila mauritiana) }\end{array}$ & mau w[12] & $\begin{array}{l}\text { Drosophila species } \\
\text { stock center; NCBI SRA }\end{array}$ & $\begin{array}{l}\text { 14021-0241.60; } \\
\text { SRX684364; } \\
\text { SRX135546 }\end{array}$ & \\
\hline $\begin{array}{l}\text { Genetic reagent } \\
\text { (Drosophila simulans }\end{array}$ & $\operatorname{sim} w[X D 1]$ & this paper & SRR8247551 & obtained from J. Coyne \\
\hline $\begin{array}{l}\text { Genetic reagent } \\
\text { (Drosophila mauritiana) }\end{array}$ & $2 P-1$ & this paper & & $\begin{array}{l}w[12], P\{w[+]=\text { Neneh2 }\} \\
P\{w[+]=4 R 1\}\end{array}$ \\
\hline $\begin{array}{l}\text { Genetic reagent } \\
\text { (Drosophila mauritiana) }\end{array}$ & $2 P-3$ & this paper & & $\begin{array}{l}\mathrm{w}[12], \mathrm{P}\{\mathrm{w}[+]=\text { Ophelia1 }\} \\
\mathrm{P}\{\mathrm{w}[+]=4 \mathrm{~J} 1\}\end{array}$ \\
\hline $\begin{array}{l}\text { Genetic reagent } \\
\text { (Drosophila mauritiana) }\end{array}$ & $2 P-4$ & this paper & & $\begin{array}{l}\mathrm{w}[12], \mathrm{P}\{\mathrm{w}[+]=4 \mathrm{~J} 1\} \\
\mathrm{P}\{\mathrm{w}[+]=2 \mathrm{~A} 1\}\end{array}$ \\
\hline $\begin{array}{l}\text { Genetic reagent } \\
\text { (Drosophila mauritiana) }\end{array}$ & $2 P-5 a$ & this paper & & $\begin{array}{l}w[12], P\{w[+]=2 A 1\}, \\
P\{w[+]=I L E A 1\}\end{array}$ \\
\hline $\begin{array}{l}\text { Genetic reagent } \\
\text { (Drosophila mauritiana) }\end{array}$ & $2 P-5 b$ & this paper & & $\begin{array}{l}w[12], P\{w[+]=2 A 1\}, \\
P\{w[+]=2 G 3\}\end{array}$ \\
\hline $\begin{array}{l}\text { Genetic reagent } \\
\text { (Drosophila mauritiana) }\end{array}$ & $2 P-6 a$ & this paper & & $\begin{array}{l}w[12], P\{w[+]=2 G 3\} \\
P\{w[+]=A 1\}\end{array}$ \\
\hline $\begin{array}{l}\text { Genetic reagent } \\
\text { (Drosophila mauritiana) }\end{array}$ & $2 P-6 b$ & this paper & & $\begin{array}{l}w[12], P\{w[+]=\mid L E A 1\} \\
P\{w[+]=A 1\}\end{array}$ \\
\hline $\begin{array}{l}\text { Genetic reagent } \\
\text { (Drosophila mauritiana) }\end{array}$ & $2 P-7$ & this paper & & $\begin{array}{l}w[12], P\{w[+]=A 1\} \\
P\{w[+]=3 L 1\}\end{array}$ \\
\hline $\begin{array}{l}\text { Genetic reagent } \\
\text { (Drosophila simulans) }\end{array}$ & YFP[175.2] & PMID:28280212 & & pBac $\{3 X P 3:: E Y F P-a t t P\}$ \\
\hline $\begin{array}{l}\text { Genetic reagent } \\
\text { (Drosophila simulans) }\end{array}$ & YFP[356.5] & PMID:28280212 & & pBac $\{3 X P 3:: E Y F P-a t t P\}$ \\
\hline $\begin{array}{l}\text { Genetic reagent } \\
\text { (Drosophila simulans) }\end{array}$ & YFP[377.31] & PMID:28280212 & & pBac $\{3 X P 3:: E Y F P-a t t P\}$ \\
\hline
\end{tabular}

Continued on next page 
Continued

\begin{tabular}{|c|c|c|c|c|}
\hline $\begin{array}{l}\text { Reagent type } \\
\text { (species) or resource }\end{array}$ & Designation & Source or reference & Identifiers & Additional information \\
\hline $\begin{array}{l}\text { Genetic reagent } \\
\text { (Drosophila simulans) }\end{array}$ & YFP[52.4] & PMID:28280212 & & pBac $\{3 X P 3:: E Y F P-a t t P\}$ \\
\hline $\begin{array}{l}\text { Genetic reagent } \\
\text { (Drosophila simulans) }\end{array}$ & YFP[277.1] & PMID:28280212 & & pBac $\{3 X P 3:: E Y F P-a t t P\}$ \\
\hline $\begin{array}{l}\text { Genetic reagent } \\
\text { (Drosophila simulans) }\end{array}$ & YFP[926.3] & PMID:28280212 & & pBac $\{3 X P 3:: E Y F P-a t t P\}$ \\
\hline $\begin{array}{l}\text { Genetic reagent } \\
\text { (Drosophila simulans) }\end{array}$ & YFP[16.3] & PMID:28280212 & & pBac\{3XP3::EYFP-attP\} \\
\hline $\begin{array}{l}\text { Genetic reagent } \\
\text { (Drosophila simulans) }\end{array}$ & YFP[360.1] & PMID:28280212 & & pBac $\{3 X P 3:: E Y F P-a t t P\}$ \\
\hline $\begin{array}{l}\text { Genetic reagent } \\
\text { (Drosophila simulans) }\end{array}$ & YFP[433.1] & PMID:28280212 & & pBac $\{3 X P 3:: E Y F P-a t t P\}$ \\
\hline $\begin{array}{l}\text { Genetic reagent } \\
\text { (Drosophila simulans) }\end{array}$ & YFP[19.1] & PMID:28280212 & & pBac $\{3 X P 3:: E Y F P-a t t P\}$ \\
\hline $\begin{array}{l}\text { Genetic reagent } \\
\text { (Drosophila simulans) }\end{array}$ & YFP[21.4] & PMID:28280212 & & pBac $\{3 X P 3:: E Y F P-a t t P\}$ \\
\hline $\begin{array}{l}\text { Genetic reagent } \\
\text { (Drosophila simulans) }\end{array}$ & YFP[458.6] & PMID:28280212 & & pBac $\{3 X P 3:: E Y F P-a t t P\}$ \\
\hline $\begin{array}{l}\text { Sequence-based } \\
\text { reagent }\end{array}$ & Dox_F_1 & this paper & & CGAAATGAGACGCTTCTGTG \\
\hline $\begin{array}{l}\text { Sequence-based } \\
\text { reagent }\end{array}$ & Dox_R_1 & this paper & & $\begin{array}{l}\text { AACCGATACCG } \\
\text { TCGTAGTTGAC }\end{array}$ \\
\hline $\begin{array}{l}\text { Sequence-based } \\
\text { reagent }\end{array}$ & MDox_F_1 & this paper & & $\begin{array}{l}\text { CCCATTTTGT } \\
\text { CCAAGGTCAC }\end{array}$ \\
\hline $\begin{array}{l}\text { Sequence-based } \\
\text { reagent }\end{array}$ & MDox_R_2 & this paper & & $\begin{array}{l}\text { AGTTCCGGTC } \\
\text { AAAGTGGTTG }\end{array}$ \\
\hline $\begin{array}{l}\text { Sequence-based } \\
\text { reagent }\end{array}$ & RpS28b_F_1 & this paper & & $\begin{array}{l}\text { TGGACAAACC } \\
\text { AGTTGTGTGG }\end{array}$ \\
\hline $\begin{array}{l}\text { Sequence-based } \\
\text { reagent }\end{array}$ & RpS28b_R_1 & this paper & & $\begin{array}{l}\text { AGGAACTCGA } \\
\text { CCTTCACCTG }\end{array}$ \\
\hline $\begin{array}{l}\text { Strain } \\
\text { (Drosophila simulans) }\end{array}$ & $\operatorname{sim} w[501]$ & PMID:22936249 & $14021-0251.011$ & \\
\hline $\begin{array}{l}\text { Strain } \\
\text { (Drosophila simulans) }\end{array}$ & md06 & NCBI SRA & SRX497551 & \\
\hline $\begin{array}{l}\text { Strain } \\
\text { (Drosophila simulans) }\end{array}$ & md15 & NCBI SRA & SRX497574 & \\
\hline $\begin{array}{l}\text { Strain } \\
\text { (Drosophila simulans) }\end{array}$ & md63 & NCBI SRA & SRX497553 & \\
\hline $\begin{array}{l}\text { Strain } \\
\text { (Drosophila simulans) }\end{array}$ & md73 & NCBI SRA & SRX497563 & \\
\hline $\begin{array}{l}\text { Strain } \\
\text { (Drosophila simulans) }\end{array}$ & md105 & NCBI SRA & SRX497558 & \\
\hline $\begin{array}{l}\text { Strain } \\
\text { (Drosophila simulans) }\end{array}$ & md199 & NCBI SRA & SRX497559 & \\
\hline $\begin{array}{l}\text { Strain } \\
\text { (Drosophila simulans) }\end{array}$ & md221 & NCBI SRA & SRX495510 & \\
\hline $\begin{array}{l}\text { Strain } \\
\text { (Drosophila simulans) }\end{array}$ & md233 & NCBI SRA & SRX495507 & \\
\hline $\begin{array}{l}\text { Strain } \\
\text { (Drosophila simulans) }\end{array}$ & md251 & NCBI SRA & SRX497557 & \\
\hline $\begin{array}{l}\text { Strain } \\
\text { (Drosophila simulans) }\end{array}$ & ns05 & NCBI SRA & SRX497560 & \\
\hline
\end{tabular}

Continued on next page 
Continued

\begin{tabular}{|c|c|c|c|c|}
\hline $\begin{array}{l}\text { Reagent type } \\
\text { (species) or resource }\end{array}$ & Designation & Source or reference & Identifiers & Additional information \\
\hline $\begin{array}{l}\text { Strain } \\
\text { (Drosophila simulans) }\end{array}$ & ns33 & NCBI SRA & SRX497575 & \\
\hline $\begin{array}{l}\text { Strain } \\
\text { (Drosophila simulans) }\end{array}$ & ns39 & NCBI SRA & SRX497562 & \\
\hline $\begin{array}{l}\text { Strain } \\
\text { (Drosophila simulans) }\end{array}$ & ns40 & NCBI SRA & SRX497556 & \\
\hline $\begin{array}{l}\text { Strain } \\
\text { (Drosophila simulans) }\end{array}$ & ns50 & NCBI SRA & SRX497571 & \\
\hline $\begin{array}{l}\text { Strain } \\
\text { (Drosophila simulans) }\end{array}$ & ns67 & NCBI SRA & SRX497565 & \\
\hline $\begin{array}{l}\text { Strain } \\
\text { (Drosophila simulans) }\end{array}$ & ns78 & NCBI SRA & SRX497573 & \\
\hline $\begin{array}{l}\text { Strain } \\
\text { (Drosophila simulans) }\end{array}$ & ns79 & NCBI SRA & SRX497576 & \\
\hline $\begin{array}{l}\text { Strain } \\
\text { (Drosophila simulans) }\end{array}$ & ns113 & NCBI SRA & SRX497572 & \\
\hline $\begin{array}{l}\text { Strain } \\
\text { (Drosophila simulans) }\end{array}$ & ns137 & NCBI SRA & SRX497561 & \\
\hline $\begin{array}{l}\text { Strain } \\
\text { (Drosophila mauritiana) }\end{array}$ & $r 12$ & NCBI SRA & SRX135546 & \\
\hline $\begin{array}{l}\text { Strain } \\
\text { (Drosophila mauritiana) }\end{array}$ & r23 & NCBI SRA & SRX688576 & \\
\hline $\begin{array}{l}\text { strain } \\
\text { (Drosophila mauritiana) }\end{array}$ & r31 & NCBI SRA & SRX688581 & \\
\hline $\begin{array}{l}\text { Strain } \\
\text { (Drosophila mauritiana) }\end{array}$ & r32 & NCBI SRA & SRX688583 & \\
\hline $\begin{array}{l}\text { Strain } \\
\text { (Drosophila mauritiana) }\end{array}$ & r39 & NCBI SRA & SRX688588 & \\
\hline $\begin{array}{l}\text { Strain } \\
\text { (Drosophila mauritiana) }\end{array}$ & r41 & NCBI SRA & SRX688609 & \\
\hline $\begin{array}{l}\text { Strain } \\
\text { (Drosophila mauritiana) }\end{array}$ & r44 & NCBI SRA & SRX688610 & \\
\hline $\begin{array}{l}\text { Strain } \\
\text { (Drosophila mauritiana) }\end{array}$ & r56 & NCBI SRA & SRX688612 & \\
\hline $\begin{array}{l}\text { Strain } \\
\text { (Drosophila mauritiana) }\end{array}$ & r61 & NCBI SRA & SRX688710 & \\
\hline $\begin{array}{l}\text { Strain } \\
\text { (Drosophila mauritiana) }\end{array}$ & r8 & NCBI SRA & SRX688712 & \\
\hline
\end{tabular}

(Drosophila mauritiana)

\section{Drosophila husbandry and genetics}

All Drosophila crosses and phenotyping were done in parallel in two locations, using standard cornmeal media (Rochester, NY) or minimal cornmeal media (Bloomington, IN) at room temperature (2325C). We constructed $D$. mauritiana ' $2 P^{\prime}$ lines that carry pairs of $X$-linked $P$-element insertions that contain the mini-white transgene $\left(P\left[w^{+}\right]\right)$(True et alo, 1996a) which serve as semi-dominant visible genetic eye-color markers and allow us to distinguish individuals carrying 0,1 or $2 P\left[w^{+}\right]$. These ' $2 P^{\prime}$ regions were then introgressed into the $D$. simulans $W^{X D 1}$ genetic background through more than 40 generations of repeated backcrossing while following the two $P\left[w^{+}\right]$insertions (Figure 1A). Each $2 P$ introgression line was then bottlenecked through a single female to eliminate segregating variation in the recombination breakpoints flanking the $2 P\left[w^{+}\right]$interval.

We performed meiotic mapping to ascertain the genetic basis of male sterility within each $2 P$ introgression by generating recombinant $1 P$ introgression genotypes (Figure $1 B$ ). $2 P\left[w^{+}\right]$females were crossed to $D$. simulans strains carrying an X-linked pBac[eYFP] transgene (Stern et al., 2017) that served as an additional visible marker. Progeny from this cross were scored for recombinant $X$ 
chromosomes carrying both $p B a c[e Y F P]$ and a single $P\left[w^{+}\right]$(1P-YFP). Recombinant $1 P$-YFP chromosomes were generated using $p B a c[e Y F P]$ markers both proximal and distal to each $2 P$ introgression. Virgin $1 P$-YFP females were individually crossed to $D$. simulans $W^{X D 1}$ males to initiate $1 P$-YFP strains. Each $1 P$-YFP $X$ chromosome was then assayed for male fertility. At least 10 individual $1 P$-YFP males of each genotype were collected 1-2 days post-eclosion and aged 3-5 days, then placed singly in a vial with three virgin $D$. simulans $w^{\mathrm{XD} 1}$ females. After 7 days, both the male and females were discarded, and all offspring emerging from the vial were counted. Additional 1P-YFP males were archived for DNA extraction.

Progeny sex ratios were calculated as the number of female offspring/total number of offspring (\% female). Males that sired fewer than five offspring were excluded from sex ratio analyses, as were genotypes with fewer than three males that sired more than four offspring. This resulted in 2538 males and 303 recombinant $1 P$-YFP chromosomes that were used to estimate progeny sex ratios; 210 recombinant $1 P$-YFP genotypes had both progeny sex ratio and sequence data.

\section{Genotyping recombinant chromosomes by sequencing}

We determined the fine-scale genetic architecture of hybrid male sterility within each introgressed region by genotyping recombinant 1 P-YFP $X$ chromosomes using multiplexed whole-genome sequencing. DNA extraction and library construction followed published methods for high-throughput sequence analysis of a large number of recombinant genotypes (Andolfatto et alo, 2011; Peluffo et al., 2015). Sequence reads were mapped to the reference genome sequence of the $D$. mauritiana stock used for mapping ( $m a u w^{12}$ ) (Garrigan et al., 2012), the genome sequence of sim $w^{X D 1}$, and the D. simulans pBac[eYFP] strains (Stern et al., 2017). Ancestry from each parent species was determined by a Hidden Markov Model (HMM) (Pinero et al., 2017; Andolfatto et al., 2011).

Genotype data and ancestry assignments were inspected for all recombinant 1 P-YFP introgression genotypes. Genotypes were excluded if there was no segment on the $\mathrm{X}$ chromosome identified by the HMM that had either a posterior probability of $D$. mauritiana parentage $>0.95$ or a posterior probability of $D$. simulans parentage $<0.05$. Genotypes with segments that had either a posterior probability of $D$. mauritiana parentage $>0.95$ or a posterior probability of $D$. simulans parentage $<0.05$ in a region that was not within the parental $2 P$ region (i.e. came from a different $2 P$ introgression) were inferred to have resulted either from mislabeling or contamination of DNA samples and were excluded from further analyses. 112 genotypes had insufficient sequence data to identify introgressions using the criteria above (or the introgression was too small to be identified). 16 genotypes showed evidence for $D$. mauritiana alleles that did not fall within the parental $2 P$ interval. Across the 439 genotypes with sufficiently high-quality sequence data for ancestry assignment, we recovered 64,373 X-linked markers. A subset of 2835 non-redundant markers were retained that delimit the extent of each 1P-YFP D. mauritiana segment. No genotype showed evidence for any autosomal $D$. mauritiana alleles (see Figure 2-figure supplement 1 for exemplars), confirming that our introgression scheme isolated $X$-linked $D$. mauritiana segments in a pure $D$. simulans autosomal genome.

\section{Quantitative trait locus analysis}

QTL analyses were done in the R/qtl package version 1.36-6. Phenotype means (fertility and progeny sex-ratio) for each introgression genotype and the 2835 non-redundant markers were used as the input data. Mean male fertility was transformed as $\log _{10}(N+1)$. Because of the large proportion of completely sterile introgression genotypes (Figure 1-figure supplement 1), a two-part model (Broman et al., 2003) was used to analyze fertility; sex-ratio was analyzed assuming a normal distribution. Significance thresholds were determined using 10,000 permutations of the data.

\section{Samples and short read alignment}

We used genome sequence data from 10 lines of $D$. mauritiana, including nine inbred wild isolates and the genome reference strain, mau $w^{12} ; 20$ lines of $D$. simulans, including 10 inbred wild isolates from Kenya, nine wild isolates from Madagascar, and the reference strain, sim $w^{501}$; and the reference strain of $D$. melanogaster. The $D$. mauritiana and $D$. simulans sequence data were reported previously (Garrigan et al., 2012; Garrigan et al., 2014; Rogers et al., 2014). SRA accessions for genome sequences are included in the key resources file. The $D$. simulans $w^{501}$ and $D$. melanogaster 
genome assemblies are available on Flybase (www.flybase.org). We performed short read alignment against the $D$. mauritiana genome assembly (version 2) using the 'aln/sampe' functions of the BWA short read aligner and default settings (Li and Durbin, 2009). Reads flanking indels were realigned using the SAMTOOLS software (Li et al., 2009). Individual BAM files were merged and sorted with SAMTOOLS.

\section{Polymorphism and divergence analyses}

Both within- and between-population summary statistics were estimated in 10-kb windows using the software package POPBAM (Garrigan, 2013). The within population summary statistics include: unbiased nucleotide diversity $\pi$ (Nei, 1987); the summary of the folded site frequency spectrum Tajima's D (Tajima, 1989); and the unweighted average pairwise value of the $r^{2}$ measure of linkage disequilibrium, $Z n S$, excluding singletons (Kelly, 1997). The between population summary statistics include: two measures of nucleotide divergence between populations, $D_{X Y}$, and net divergence, $D_{A}$ (Nei, 1987); the ratio of the minimum between-population nucleotide distance to the average, $G_{\min }$ (Geneva et al., 2015); and the fixation index, $F_{S T}$ (Wright, 1951). From a total of 11,083 scanned $10-\mathrm{kb}$ windows, we only analyzed windows for which at least $50 \%$ of aligned sites passed the default quality filters (minimum read coverage 3 , minimum rms mapping quality 25 , minimum SNP quality 25 , minimum map quality 13 , minimum base quality 13) in POPBAM, which resulted in a final alignment for 10,443 scanned 10-kb windows. POPBAM output was formatted for use in the R statistical computing environment using the package, POPBAMTools (Geneva, 2014). All statistics and data visualization were done in $\mathrm{R}$ ( $R$ Development Core Team, 2013).

\section{Identification of introgressed regions}

We used the $G_{\min }$ statistic (Geneva et al., 2015) to scan the genome for haplotypes that have recent common ancestry between $D$. simulans and $D$. mauritiana. $G_{\min }$ is defined as the ratio of the minimum number of nucleotide differences per aligned site between sequences from different populations to the average number of nucleotide differences per aligned site between populations. The $G_{\min }$ statistic was calculated in 10-kb intervals across each major chromosome arm using the same quality filtering criteria used for all other summary statistics. From these values, we estimated the probability of the observed $G_{\min }$ under a model of allopatric divergence, conditioned on the divergence time. For each $10-\mathrm{kb}$ genomic interval, the significance of the observed $G_{\min }$ value was tested via Monte Carlo coalescent simulation of that $10-\mathrm{kb}$ window with two populations diverging in allopatry with all mutations assumed to be neutral. Simulations were performed using msmove (Geneva, 2017), which is based on the coalescent simulation software ms (Hudson, 2002), modified to track and report the presence of introgressed genealogies. The arguments of msmove are identical to those of $\mathrm{ms}$ and for all simulations we used the following command (msmove 30 10000 t $\theta$-r $\rho 10001$-I 21020 -ej 0.61 1 2). We assumed a population divergence time of $1.21 \times 2_{\text {Nsim }}$ generations before the present, in which $N_{\text {sim }}$ is the current estimated effective population size of $D$. simulans (Garrigan et al., 2012). In the simulations, the observed local value of $D_{X Y}$ was used to determine the neutral population mutation rate $(\theta)$ for that $10-k b$ interval. To account for uncertainty in local population recombination rate, for each simulated replicate, a rate was drawn from a normally distributed prior (truncated at zero) with the mean estimated from genetically determined crossover frequencies (True et al., 1996a) for that window, and variance equal to the variance of crossover estimates for the entire chromosome arm. The empirical crossover rate estimates were converted from $\mathrm{cM}$ to $\rho$ (the population crossover rate, $4 N_{\text {sim }} c$ ) by assuming $N_{\text {sim }} \approx 10^{6}$. The effective population sizes of both species were assumed to be equal and constant. For each 10-kb interval, $10^{5}$ simulated replicates were generated and the probability of the observed $G_{\min }$ value was estimated from the simulated cumulative density. To identify putatively introgressed haplotypes, we used a significance threshold of $\mathrm{p} \leq 0.001$ from the simulations, which yields a proportion of null tests of 0.982 and a false discovery rate of $5 \%$. To infer the full length of any putative introgressions $>10$ $\mathrm{kb}$, we identified runs of contiguous (or semi-contiguous) $10-k b$ windows with significant $G_{\min }$ values $(p \leq 0.001)$. We also assessed the distribution of shared derived variants using the four-population test, summarized by Patterson's D statistic (Green et al., 2010). Variants were generated using POPBAM default parameters and used to calculate Patterson's $D$ across chromosome arms using customized perl scripts. For $D$ statistic calculations, we assumed the tree structure $(((D$. sechellia, $D$. 
simulans), D. mauritiana), D. melanogaster) for (((P1,P2),P3),O), and used the population frequencies of SNPs to compute probabilistic contributions of individual sites to counts of 'ABBA' and 'BABA' site types (Green et al., 2010; Durand et al., 2011). Finally, we estimated maximum likelihood phylogenies for each of the putative introgression intervals using RAxML v. 8.1.1 (Stamatakis, 2014).

\section{Genotyping the Winters sex ratio genes}

We extracted genomic DNA from single male flies using the Qiagen DNeasy Blood and Tissue Kit. The meiotic drive genes of the Winters sex ratio system (Tao et al., 2007a), Dox and MDox, were PCR-amplified as previously described (Kingan et al., 2010). To assay the presence or absence of the Dox and MDox gene insertions, the amplicons for the Dox and MDox regions were digested with the Styl and Stul restriction enzymes (NEB), respectively. The digests were run on a $1 \%$ agarose gel stained with $\mathrm{EtBr}$ and the band size was estimated using the GeneRuler $1 \mathrm{~kb}$ plus ladder (Thermo Scientific). For both genes, only haplotypes containing the gene insertions have restriction sites as confirmed by samples with known genotypes (Kingan et al., 2010).

\section{Quantitative PCR for Dox/MDox expression in fly testes}

We assayed expression of the Dox and MDox genes in testes from D. simulans strain MD63 and D. mauritiana strain mau $w^{12}$ using quantitative PCR. Total RNA was extracted from the dissected testes of 5-10 day old flies using the Nucleospin RNA XS kit (Macherey-Nagel, Germany), and cDNA was synthesized with poly dT oligos and random hexamers using Superscript III RT cDNA synthesis kit (Invitrogen, CA). qPCR assays were performed on a BioRad Real-time PCR machine using the cycling conditions: $95^{\circ} \mathrm{C}$ for 3 mins.; 40 cycles of $95^{\circ} \mathrm{C}$ for $10 \mathrm{~s}, 58^{\circ} \mathrm{C}$ for $30 \mathrm{~s}$, and $72^{\circ} \mathrm{C}$ for $30 \mathrm{~s}$. The primer sequences used for qPCR are provided in Supplementary file 3.

\section{Acknowledgements}

The authors thank Brian Calvi for generously providing laboratory space and Shelby Biel, Ally Shambaugh, and Amanda Meiklejohn for assistance with fertility assays, Cara Brand for calculation of $D$. mauritiana recombination rates, and Peter Andolfatto and Kevin Thornton for sharing D. simulans genome sequence data.

\section{Additional information}

\begin{tabular}{lll}
$\begin{array}{ll}\text { Funding } \\
\text { Funder }\end{array}$ & Grant reference number & Author \\
\hline $\begin{array}{lll}\text { National Institute of General } \\
\text { Medical Sciences }\end{array}$ & 1R01OD010548-01A1 & $\begin{array}{l}\text { Daniel Garrigan } \\
\text { Daven Presgraves }\end{array}$ \\
\hline University of Nebraska-Lincoln & & Colin Meiklejohn \\
\hline University of Rochester & & $\begin{array}{l}\text { Daniel Garrigan } \\
\text { Daven Presgraves }\end{array}$ \\
\hline National Science Foundation & DEB-0839348 & Colin Meiklejohn \\
\hline $\begin{array}{l}\text { National Institute of General } \\
\text { Medical Sciences }\end{array}$ & 1R01GM123194-01A1 & $\begin{array}{l}\text { Colin Meiklejohn } \\
\text { Daven Presgraves }\end{array}$ \\
\hline
\end{tabular}

The funders had no role in study design, data collection and interpretation, or the decision to submit the work for publication.

\section{Author contributions}

Colin D Meiklejohn, Conceptualization, Resources, Formal analysis, Supervision, Funding acquisition, Investigation, Visualization, Methodology, Writing-original draft, Project administration, Writingreview and editing; Emily L Landeen, Conceptualization, Supervision, Investigation, Writing-review and editing; Kathleen E Gordon, Investigation, Writing-review and editing; Thomas Rzatkiewicz, Investigation; Sarah B Kingan, Conceptualization, Software, Formal analysis, Supervision, Investigation, Visualization, Writing-original draft, Writing-review and editing; Anthony J Geneva, 
Software, Formal analysis, Methodology, Writing-review and editing; Jeffrey P Vedanayagam, Data curation, Formal analysis, Writing—review and editing; Christina A Muirhead, Formal analysis; Daniel Garrigan, Conceptualization, Resources, Data curation, Software, Formal analysis, Supervision, Funding acquisition, Visualization, Methodology, Writing—original draft, Project administration, Writingreview and editing; David L Stern, Resources, Software, Formal analysis, Investigation, Methodology, Writing-review and editing; Daven C Presgraves, Conceptualization, Resources, Formal analysis, Supervision, Funding acquisition, Investigation, Methodology, Writing—original draft, Project administration, Writing-review and editing

Author ORCIDs

Colin D Meiklejohn (iD https://orcid.org/0000-0003-2708-8316

Daniel Garrigan (iD http://orcid.org/0000-0002-7000-6788

David L Stern (iD http://orcid.org/0000-0002-1847-6483

Decision letter and Author response

Decision letter https://doi.org/10.7554/eLife.35468.048

Author response https://doi.org/10.7554/eLife.35468.049

\section{Additional files}

Supplementary files

- Supplementary file 1. $G_{\min }$ scan identifies forty-eight interspecific introgressions.

DOI: https://doi.org/10.7554/eLife.35468.029

- Supplementary file 2. Genotype of samples at the Dox and MDox genes.

DOl: https://doi.org/10.7554/eLife.35468.030

- Supplementary file 3. Primers used in RT-PCR to assay expression of MDox, Dox, and a control gene (RpS28b).

DOI: https://doi.org/10.7554/eLife.35468.031

- Transparent reporting form

DOl: https://doi.org/10.7554/eLife.35468.032

Data availability

Sequence data is available via the NCBI Sequence Read Archive (accession number: SRR8247551). Phenotype data have been submitted to Dryad (DOI: https://doi.org/10.5061/dryad.4qn4s47).

The following datasets were generated:

\begin{tabular}{|c|c|c|c|c|}
\hline Author(s) & Year & Dataset title & Dataset URL & $\begin{array}{l}\text { Database and } \\
\text { Identifier }\end{array}$ \\
\hline $\begin{array}{l}\text { Colin Meiklejohn, } \\
\text { Daven Presgraves, } \\
\text { David L Stern }\end{array}$ & 2018 & $\begin{array}{l}\text { Sequence data from Gene flow } \\
\text { mediates the role of sex } \\
\text { chromosome meiotic drive during } \\
\text { complex speciation }\end{array}$ & $\begin{array}{l}\text { https://www.ncbi.nlm. } \\
\text { nih.gov/sra/?term= } \\
\text { SRR8247551 }\end{array}$ & $\begin{array}{l}\text { NCBI Sequence Read } \\
\text { Archive, SRR8247551 }\end{array}$ \\
\hline $\begin{array}{l}\text { Meiklejohn CD, } \\
\text { Landeen EL, Pre- } \\
\text { sgraves DC }\end{array}$ & 2018 & $\begin{array}{l}\text { Gene flow mediates the role of sex } \\
\text { chromosome meiotic drive during } \\
\text { complex speciation }\end{array}$ & $\begin{array}{l}\text { https://dx.doi.org/10. } \\
\text { 5061/dryad.4qn4s47 }\end{array}$ & $\begin{array}{l}\text { Dryad Digital } \\
\text { Repository, } 10.5061 / \\
\text { dryad.4qn4s47 }\end{array}$ \\
\hline
\end{tabular}

The following previously published datasets were used:

\begin{tabular}{|c|c|c|c|c|}
\hline Author(s) & Year & Dataset title & Dataset URL & $\begin{array}{l}\text { Database and } \\
\text { Identifier }\end{array}$ \\
\hline $\begin{array}{l}\text { Garrigan D, Kingan } \\
\text { SB, Geneva AJ, } \\
\text { Vedanayagam JP, } \\
\text { Presgraves DC }\end{array}$ & 2014 & $\begin{array}{l}\text { Drosophila mauritiana genome } \\
\text { sequencing }\end{array}$ & $\begin{array}{l}\text { https://www.ncbi.nlm. } \\
\text { nih.gov/bioproject/ } \\
\text { PRJNA158675 }\end{array}$ & $\begin{array}{l}\text { NCBI BioProject, } \\
\text { PRJNA158675 }\end{array}$ \\
\hline $\begin{array}{l}\text { Rogers RL, Cridland } \\
\text { JM, Shao L, Hu TT, } \\
\text { Andolfatto P, } \\
\text { Thornton KR }\end{array}$ & 2015 & $\begin{array}{l}\text { Tandem Duplications and the Limits } \\
\text { of Natural Selection in Drosophila } \\
\text { yakuba and Drosophila simulans }\end{array}$ & $\begin{array}{l}\text { https://www.ncbi.nlm. } \\
\text { nih.gov/sra/?term= } \\
\text { SRP040290 }\end{array}$ & $\begin{array}{l}\text { NCBI Sequence Read } \\
\text { Archive, SRP040 } \\
290\end{array}$ \\
\hline
\end{tabular}


Rogers RL, Cridland 2015 JM, Shao L, HuTT, Andolfatto $P$,

Thornton KR
Tandem Duplications and the Limits https://www.ncbi.nlm. of Natural Selection in Drosophila yakuba and Drosophila simulans
NCBI Sequence Read Archive, SRP029453

\section{References}

Andolfatto P, Davison D, Erezyilmaz D, Hu TT, Mast J, Sunayama-Morita T, Stern DL. 2011. Multiplexed shotgun genotyping for rapid and efficient genetic mapping. Genome Research 21:610-617. DOI: https://doi.org/10. 1101/gr.115402.110, PMID: 21233398

Ballard JW. 2000a. Comparative genomics of mitochondrial DNA in members of the Drosophila melanogaster subgroup. Journal of Molecular Evolution 51:48-63. DOI: https://doi.org/10.1007/s002390010066, PMID: 10 903372

Ballard JW. 2000b. When one is not enough: introgression of mitochondrial DNA in Drosophila. Molecular Biology and Evolution 17:1126-1130. DOI: https://doi.org/10.1093/oxfordjournals.molbev.a026394, PMID: 10 889226

Ballard JW. 2004. Sequential evolution of a symbiont inferred from the host: Wolbachia and Drosophila simulans. Molecular Biology and Evolution 21:428-442. DOI: https://doi.org/10.1093/molbev/msh028, PMID: 14660690

Barton N, Bengtsson BO. 1986. The barrier to genetic exchange between hybridising populations. Heredity 57: 357-376. DOI: https://doi.org/10.1038/hdy.1986.135, PMID: 3804765

Baudry E, Derome N, Huet M, Veuille M. 2006. Contrasted polymorphism patterns in a large sample of populations from the evolutionary genetics model Drosophila simulans. Genetics 173:759-767. DOI: https:// doi.org/10.1534/genetics.105.046250, PMID: 16510794

Begun DJ, Holloway AK, Stevens K, Hillier LW, Poh YP, Hahn MW, Nista PM, Jones CD, Kern AD, Dewey CN, Pachter L, Myers E, Langley CH. 2007. Population genomics: whole-genome analysis of polymorphism and divergence in Drosophila simulans. PLOS Biology 5:e310. DOI: https://doi.org/10.1371/journal.pbio.0050310, PMID: 17988176

Bengtsson BO. 1985. The flow of genes through a genetic barrier. In: Greenwood P. J, Harvey P. H, Slatkin M (Eds). Evolution: Essays in Honour of John Maynard Smith. Cambridge: Cambridge University Press.

Broman KW, Wu H, Sen S, Churchill GA. 2003. R/qtl: QTL mapping in experimental crosses. Bioinformatics 19: 889-890. DOI: https://doi.org/10.1093/bioinformatics/btg112, PMID: 12724300

Charlesworth B, Coyne JA, Barton NH. 1987. The relative rates of evolution of sex chromosomes and autosomes. The American Naturalist 130:113-146. DOI: https://doi.org/10.1086/284701

Coyne JA, Orr HA. 1989. Two rules of speciation. In: Otte D, Endler J (Eds). Speciation and Its Consequences. Sunderland, MA: Sinauer Associates.

Coyne JA. 1992a. Genetics and speciation. Nature 355:511-515. DOI: https://doi.org/10.1038/355511a0, PMID: 1741030

Coyne JA. 1992b. Genetics of sexual isolation in females of the Drosophila simulans species complex. Genetical Research 60:25-31. DOI: https://doi.org/10.1017/S0016672300030639, PMID: 1452013

Coyne JA, Orr HA. 2004. Speciation. Sunderland, Massachusetts: Sinauer.

Crespi B, Nosil P. 2013. Conflictual speciation: species formation via genomic conflict. Trends in Ecology \& Evolution 28:48-57. DOI: https://doi.org/10.1016/j.tree.2012.08.015, PMID: 22995895

David J, Mcevey SF, Solignac M, Tsacas L. 1989. Drosophila communities on Mauritius and ecological niche of $D$. mauritiana (Diptera, Drosophilidae). Journal of African Zoology 103:107-116.

Dean MD, Ballard JW. 2004. Linking phylogenetics with population genetics to reconstruct the geographic origin of a species. Molecular Phylogenetics and Evolution 32:998-1009. DOI: https://doi.org/10.1016/j.ympev.2004. 03.013, PMID: 15288072

Durand EY, Patterson N, Reich D, Slatkin M. 2011. Testing for ancient admixture between closely related populations. Molecular Biology and Evolution 28:2239-2252. DOl: https://doi.org/10.1093/molbev/msr048, PMID: 21325092

Frank SA. 1991. Divergence of meiotic drive-suppression systems as an explanation for sex-biased hybrid sterility and inviability. Evolution; International Journal of Organic Evolution 45:262-267. DOI: https://doi.org/10.1111/ j.1558-5646.1991.tb04401.x, PMID: 28567880

Garrigan D, Kingan SB, Geneva AJ, Andolfatto P, Clark AG, Thornton KR, Presgraves DC. 2012. Genome sequencing reveals complex speciation in the Drosophila simulans clade. Genome Research 22:1499-1511. DOI: https://doi.org/10.1101/gr.130922.111, PMID: 22534282

Garrigan D. 2013. POPBAM: tools for evolutionary analysis of short read sequence alignments. Evolutionary Bioinformatics 9:EBO.S12751. DOI: https://doi.org/10.4137/EBO.S12751

Garrigan D, Kingan SB, Geneva AJ, Vedanayagam JP, Presgraves DC. 2014. Genome diversity and divergence in Drosophila mauritiana: multiple signatures of faster X evolution. Genome Biology and Evolution 6:2444-2458. DOI: https://doi.org/10.1093/gbe/evu198, PMID: 25193308

Geneva AJ. 2014. POPBAMTools. GitHub. 8129124. https://github.com/geneva/POPBAMTools

Geneva AJ, Muirhead CA, Kingan SB, Garrigan D. 2015. A new method to scan genomes for introgression in a secondary contact model. PLOS ONE 10:e0118621. DOI: https://doi.org/10.1371/journal.pone.0118621, PMID: 25874895

Geneva AJ. 2017. msmove. GitHub. dab4d4d. https://github.com/geneva/msmove 
Good JM, Dean MD, Nachman MW. 2008. A complex genetic basis to X-linked hybrid male sterility between two species of house mice. Genetics 179:2213-2228. DOI: https://doi.org/10.1534/genetics.107.085340, PMID: 186 89897

Green RE, Krause J, Briggs AW, Maricic T, Stenzel U, Kircher M, Patterson N, Li H, Zhai W, Fritz MH, Hansen NF, Durand EY, Malaspinas AS, Jensen JD, Marques-Bonet T, Alkan C, Prüfer K, Meyer M, Burbano HA, Good JM, et al. 2010. A draft sequence of the Neandertal genome. Science 328:710-722. DOI: https://doi.org/10.1126/ science.1188021, PMID: 20448178

Haldane JBS. 1922. Sex ratio and unisexual sterility in hybrid animals. Journal of Genetics 12:101-109. DOI: https://doi.org/10.1007/BF02983075

Helleu Q, Gérard PR, Dubruille R, Ogereau D, Prud'homme B, Loppin B, Montchamp-Moreau C. 2016. Rapid evolution of a Y-chromosome heterochromatin protein underlies sex chromosome meiotic drive. PNAS 113: 4110-4115. DOI: https://doi.org/10.1073/pnas.1519332113, PMID: 26979956

Hey J, Kliman RM. 1993. Population genetics and phylogenetics of DNA sequence variation at multiple loci within the Drosophila melanogaster species complex. Molecular biology and evolution 10:804-822. DOI: https://doi.org/10.1093/oxfordjournals.molbev.a040044, PMID: 8355601

Höllinger I, Hermisson J. 2017. Bounds to parapatric speciation: A Dobzhansky-Muller incompatibility model involving autosomes, $X$ chromosomes, and mitochondria. Evolution 71:1366-1380. DOI: https://doi.org/10 1111/evo.13223, PMID: 28272742

Hu TT, Eisen MB, Thornton KR, Andolfatto P. 2013. A second-generation assembly of the Drosophila simulans genome provides new insights into patterns of lineage-specific divergence. Genome research 23:89-98. DOI: https://doi.org/10.1101/gr.141689.112, PMID: 22936249

Hudson RR, Coyne JA. 2002. Mathematical consequences of the genealogical species concept. Evolution 56: 1557-1565. DOI: https://doi.org/10.1111/j.0014-3820.2002.tb01467.x, PMID: 12353748

Hudson RR. 2002. Generating samples under a Wright-Fisher neutral model of genetic variation. Bioinformatics 18:337-338. DOI: https://doi.org/10.1093/bioinformatics/18.2.337, PMID: 11847089

Hurst LD, Pomiankowski A. 1991. Causes of sex ratio bias may account for unisexual sterility in hybrids: a new explanation of Haldane's rule and related phenomena. Genetics 128:841-858. PMID: 1916248

Jaenike J. 2001. Sex chromosome meiotic drive. Annual Review of Ecology and Systematics 32:25-49.

DOI: https://doi.org/10.1146/annurev.ecolsys.32.081501.113958

Kelly JK. 1997. A test of neutrality based on interlocus associations. Genetics 146:1197-1206. PMID: 9215920

Kingan SB, Garrigan D, Hartl DL. 2010. Recurrent selection on the Winters sex-ratio genes in Drosophila simulans. Genetics 184:253-265. DOI: https://doi.org/10.1534/genetics.109.109587, PMID: 19897749

Kliman RM, Andolfatto P, Coyne JA, Depaulis F, Kreitman M, Berry AJ, McCarter J, Wakeley J, Hey J. 2000. The population genetics of the origin and divergence of the Drosophila simulans complex species. Genetics 156: 1913-1931. PMID: 11102384

Kopp A. 2006. Basal relationships in the Drosophila melanogaster species group. Molecular Phylogenetics and Evolution 39:787-798. DOI: https://doi.org/10.1016/j.ympev.2006.01.029, PMID: 16527496

Lachaise D, David JR, Lemeunier F, Tsacas L, Ashburner M. 1986. The reproductive relationships of Drosophila sechellia with D. mauritiana, D. simulans, and D. melanogaster from the Afrotropical region. Evolution 1986: 262-271. DOI: https://doi.org/10.1111/j.1558-5646.1986.tb00468.x

Lachaise D, Cariou M-L, David JR, Lemeunier F, Tsacas L, Ashburner M. 1988. Historical biogeography of the Drosophila melanogaster species subgroup. Evolutionary Biology 22:159-225. DOI: https://doi.org/10.1007/ 978-1-4613-0931-4_4

Laurie CC. 1997. The weaker sex is heterogametic: 75 years of Haldane's rule. Genetics 147:937-951. PMID: 93 83043

Legrand D, Chenel T, Campagne C, Lachaise D, Cariou ML. 2011. Inter-island divergence within Drosophila mauritiana, a species of the $D$. simulans complex: Past history and/or speciation in progress? Molecular Ecology 20:2787-2804. DOI: https://doi.org/10.1111/j.1365-294X.2011.05127.x, PMID: 21599771

Li H, Durbin R. 2009. Fast and accurate short read alignment with Burrows-Wheeler transform. Bioinformatics 25: 1754-1760. DOI: https://doi.org/10.1093/bioinformatics/btp324, PMID: 19451168

Li H, Handsaker B, Wysoker A, Fennell T, Ruan J, Homer N, Marth G, Abecasis G, Durbin R, 1000 Genome Project Data Processing Subgroup. 2009. The sequence alignment/map format and SAMtools. Bioinformatics 25:2078-2079. DOI: https://doi.org/10.1093/bioinformatics/btp352, PMID: 19505943

Lifschytz E, Lindsley DL. 1972. The role of X-Chromosome inactivation during spermatogenesis. PNAS 69:182186. DOI: https://doi.org/10.1073/pnas.69.1.182

Lindholm AK, Dyer KA, Firman RC, Fishman L, Forstmeier W, Holman L, Johannesson H, Knief U, Kokko H, Larracuente AM, Manser A, Montchamp-Moreau C, Petrosyan VG, Pomiankowski A, Presgraves DC, Safronova LD, Sutter A, Unckless RL, Verspoor RL, Wedell N, et al. 2016. The Ecology and Evolutionary Dynamics of Meiotic Drive. Trends in Ecology \& Evolution 31:315-326. DOI: https://doi.org/10.1016/j.tree.2016.02.001, PMID: 26920473

Macaya-Sanz D, Suter L, Joseph J, Barbará T, Alba N, González-Martínez SC, Widmer A, Lexer C. 2011. Genetic analysis of post-mating reproductive barriers in hybridizing European Populus species. Heredity 107:478-486. DOI: https://doi.org/10.1038/hdy.2011.35, PMID: 21587301

Maside XR, Barral JP, Naveira HF. 1998. Hidden effects of X chromosome introgressions on spermatogenesis in Drosophila simulans $\times$ D. mauritiana hybrids unveiled by interactions among minor genetic factors. Genetics 150:745-754. PMID: 9755205 
Masly JP, Presgraves DC. 2007. High-resolution genome-wide dissection of the two rules of speciation in Drosophila. PLOS Biology 5:e243. DOI: https://doi.org/10.1371/journal.pbio.0050243, PMID: 17850182

McDermott SR, Kliman RM. 2008. Estimation of isolation times of the island species in the Drosophila simulans complex from multilocus DNA sequence data. PLoS One 3:e2442. DOI: https://doi.org/10.1371/journal.pone. 0002442, PMID: 18560591

Meiklejohn CD, Tao Y. 2010. Genetic conflict and sex chromosome evolution. Trends in Ecology \& Evolution 25: 215-223. DOI: https://doi.org/10.1016/j.tree.2009.10.005, PMID: 19931208

Moehring AJ, Teeter KC, Noor MA. 2007. Genome-wide patterns of expression in Drosophila pure species and hybrid males. II. Examination of multiple-species hybridizations, platforms, and life cycle stages. Molecular Biology and Evolution 24:137-145. DOl: https://doi.org/10.1093/molbev/ms/142, PMID: 17032727

Montchamp-Moreau C, Ogereau D, Chaminade N, Colard A, Aulard S. 2006. Organization of the sex-ratio meiotic drive region in Drosophila simulans. Genetics 174:1365-1371. DOI: https://doi.org/10.1534/genetics. 105.051755, PMID: 16387875

Moyle LC, Muir CD, Han MV, Hahn MW. 2010. The contribution of gene movement to the "two rules of speciation". Evolution 64:1541-1557. DOl: https://doi.org/10.1111/j.1558-5646.2010.00990.x, PMID: 20298429

Muirhead CA, Presgraves DC. 2016. Hybrid Incompatibilities, Local Adaptation, and the Genomic Distribution of Natural Introgression between Species. The American Naturalist 187:249-261. DOI: https://doi.org/10.1086/ 684583, PMID: 26807751

Nei M, Li WH. 1979. Mathematical model for studying genetic variation in terms of restriction endonucleases. PNAS 76:5269-5273. DOI: https://doi.org/10.1073/pnas.76.10.5269, PMID: 291943

Nei M. 1987. Molecular Evolutionary Genetics, New York. Columbia University Press.

Nolte V, Pandey RV, Kofler R, Schlötterer C. 2013. Genome-wide patterns of natural variation reveal strong selective sweeps and ongoing genomic conflict in Drosophila mauritiana. Genome Research 23:99-110. DOI: https://doi.org/10.1101/gr.139873.112, PMID: 23051690

Orr HA. 1997. Haldane's rule. Annual Review of Ecology and Systematics 28:195-218. DOI: https://doi.org/10. 1146/annurev.ecolsys.28.1.195

Orr HA, Irving S. 2005. Segregation distortion in hybrids between the Bogota and USA subspecies of Drosophila pseudoobscura. Genetics 169:671-682. DOI: https://doi.org/10.1534/genetics.104.033274, PMID: 15654115

Peluffo AE, Nuez I, Debat V, Savisaar R, Stern DL, Orgogozo V. 2015. A Major Locus Controls a Genital Shape Difference Involved in Reproductive Isolation Between Drosophila yakuba and Drosophila santomea. G3\&amp; \#58; Genes/Genomes/Genetics 5:2893-2901. DOI: https://doi.org/10.1534/g3.115.023481, PMID: 26511499

Petry D. 1983. The effect on neutral gene flow of selection at a linked locus. Theoretical Population Biology 23: 300-313. DOI: https://doi.org/10.1016/0040-5809(83)90020-5, PMID: 6623407

Phadnis N, Orr HA. 2009. A single gene causes both male sterility and segregation distortion in Drosophila hybrids. Science 323:376-379. DOI: https://doi.org/10.1126/science.1163934, PMID: 19074311

Pinero G, Reilly P, Stern D, Hu T, Parsons L. 2017. MSG: Multiplexed Shotgun Genotyping. Github. b2dcddb. https://github.com/YourePrettyGood/msg

Presgraves DC. 2002. Patterns of postzygotic isolation in Lepidoptera. Evolution 56:1168-1183. DOI: https://doi. org/10.1111/j.0014-3820.2002.tb01430.x, PMID: 12144018

Presgraves DC. 2008. Sex chromosomes and speciation in Drosophila. Trends in Genetics 24:336-343. DOI: https://doi.org/10.1016/j.tig.2008.04.007, PMID: 18514967

Presgraves DC. 2018. Evaluating genomic signatures of "the large X-effect" during complex speciation. Molecular Ecology 27:3822-3830. DOI: https://doi.org/10.1111/mec.14777, PMID: 29940087

Price CS. 1997. Conspecific sperm precedence in Drosophila. Nature 388:663-666. DOI: https://doi.org/10. 1038/41753, PMID: 9262398

Price TD, Bouvier MM. 2002. The evolution of F1 postzygotic incompatibilities in birds. Evolution 56:2083-2089. DOI: https://doi.org/10.1111/j.0014-3820.2002.tb00133.x, PMID: 12449494

R Development Core Team. 2013. R: A Language and Environment for Statistical Computing. Vienna, Austria: R Foundation for Statistical Computing

Rogers RL, Cridland JM, Shao L, Hu TT, Andolfatto P, Thornton KR. 2014. Landscape of standing variation for tandem duplications in Drosophila yakuba and Drosophila simulans. Molecular Biology and Evolution 31:17501766. DOI: https://doi.org/10.1093/molbev/msu124, PMID: 24710518

Rosenzweig BK, Pease JB, Besansky NJ, Hahn MW. 2016. Powerful methods for detecting introgressed regions from population genomic data. Molecular Ecology 25:2387-2397. DOI: https://doi.org/10.1111/mec.13610, PMID: 26945783

Sankararaman S, Mallick S, Dannemann M, Prüfer K, Kelso J, Pääbo S, Patterson N, Reich D. 2014. The genomic landscape of Neanderthal ancestry in present-day humans. Nature 507:354-357. DOI: https://doi.org/10.1038/ nature12961, PMID: 24476815

Sankararaman S, Mallick S, Patterson N, Reich D. 2016. The Combined Landscape of Denisovan and Neanderthal Ancestry in Present-Day Humans. Current Biology 26:1241-1247. DOl: https://doi.org/10.1016/j. cub.2016.03.037, PMID: 27032491

Satta Y, Toyohara N, Ohtaka C, Tatsuno Y, Watanabe TK, Matsuura ET, Chigusa SI, Takahata N. 1988. Dubious maternal inheritance of mitochondrial DNA inD. simulans and evolution of D. mauritiana. Genetical Research 52:1-6. DOI: https://doi.org/10.1017/S0016672300027245

Satta Y, Takahata N. 1990. Evolution of Drosophila mitochondrial DNA and the history of the melanogaster subgroup. PNAS 87:9558-9562. DOI: https://doi.org/10.1073/pnas.87.24.9558, PMID: 2124697 
Schrider DR, Ayroles J, Matute DR, Kern AD. 2018. Supervised machine learning reveals introgressed loci in the genomes of Drosophila simulans and D. sechellia. PLOS Genetics 14:e1007341. DOI: https://doi.org/10.1371/ journal.pgen.1007341, PMID: 29684059

Seehausen O, Butlin RK, Keller I, Wagner CE, Boughman JW, Hohenlohe PA, Peichel CL, Saetre GP, Bank C, Brännström A, Brelsford A, Clarkson CS, Eroukhmanoff F, Feder JL, Fischer MC, Foote AD, Franchini P, Jiggins CD, Jones FC, Lindholm AK, et al. 2014. Genomics and the origin of species. Nature Reviews Genetics 15:176192. DOI: https://doi.org/10.1038/nrg3644, PMID: 24535286

Slatkin M. 1993. Isolation by distance in equilibrium and non-equilibrium populations. Evolution 47:264-279. DOI: https://doi.org/10.1111/j.1558-5646.1993.tb01215.x

Solignac M, Monnerot M, Mounolou JC. 1986. Mitochondrial DNA evolution in the melanogaster species subgroup of Drosophila. Journal of Molecular Evolution 23:31-40. DOI: https://doi.org/10.1007/BF02100996, PMID: 3009834

Solignac M, Monnerot M. 1986. Race formation, speciation, and introgression within Drosophila simulans, $D$. mauritiana, and D. sechellia inferred from mitochondrial DNA analysis. Evolution 40:531-539. DOI: https://doi. org/10.1111/j.1558-5646.1986.tb00505.x, PMID: 28556334

Stamatakis A. 2014. RAxML version 8: a tool for phylogenetic analysis and post-analysis of large phylogenies. Bioinformatics 30:1312-1313. DOI: https://doi.org/10.1093/bioinformatics/btu033, PMID: 24451623

Stern DL, Crocker J, Ding Y, Frankel N, Kappes G, Kim E, Kuzmickas R, Lemire A, Mast JD, Picard S. 2017. Genetic and Transgenic Reagents for Drosophila simulans, D. mauritiana, D. yakuba, D. santomea, and D. virilis. G3: Genes/Genomes/Genetics 7:1339-1347. DOI: https://doi.org/10.1534/g3.116.038885, PMID: 28280212

Tajima F. 1989. Statistical method for testing the neutral mutation hypothesis by DNA polymorphism. Genetics 123:585-595. PMID: 2513255

Tao Y, Hartl DL, Laurie CC. 2001. Sex-ratio segregation distortion associated with reproductive isolation in Drosophila. PNAS 98:13183-13188. DOI: https://doi.org/10.1073/pnas.231478798, PMID: 11687638

Tao Y, Chen S, Hartl DL, Laurie CC. 2003. Genetic dissection of hybrid incompatibilities between Drosophila simulans and D. mauritiana. I. Differential accumulation of hybrid male sterility effects on the $\mathrm{X}$ and autosomes. Genetics 164:1383-1397. PMID: 12930747

Tao Y, Hartl DL. 2003. Genetic dissection of hybrid incompatibilities between Drosophila simulans and D. mauritiana. III. Heterogeneous accumulation of hybrid incompatibilities, degree of dominance, and implications for Haldane's rule. Evolution 57:2580-2589. PMID: 14686533

Tao Y, Araripe L, Kingan SB, Ke Y, Xiao H, Hartl DL. 2007a. A sex-ratio meiotic drive system in Drosophila simulans. II: an X-linked distorter. PLOS Biology 5:e293. DOI: https://doi.org/10.1371/journal.pbio.0050293, PMID: 17988173

Tao Y, Masly JP, Araripe L, Ke Y, Hartl DL. 2007b. A sex-ratio meiotic drive system in Drosophila simulans. I: an autosomal suppressor. PLOS Biology 5:e292. DOI: https://doi.org/10.1371/journal.pbio.0050292, PMID: 17 988172

True JR, Mercer JM, Laurie CC. 1996a. Differences in crossover frequency and distribution among three sibling species of Drosophila. Genetics 142:507-523. PMID: 8852849

True JR, Weir BS, Laurie CC. 1996b. A genome-wide survey of hybrid incompatibility factors by the introgression of marked segments of Drosophila mauritiana chromosomes into Drosophila simulans. Genetics 142:819-837. PMID: 8849890

Turissini DA, Matute DR. 2017. Fine scale mapping of genomic introgressions within the Drosophila yakuba clade. PLOS Genetics 13:e1006971. DOI: https://doi.org/10.1371/journal.pgen.1006971, PMID: 28873409

Wright S. 1951. The genetical structure of populations. Annals of Eugenics 15:323-354. DOl: https://doi.org/10. 1111/j.1469-1809.1949.tb02451.x, PMID: 24540312

Wu Cl, Davis AW. 1993. Evolution of postmating reproductive isolation: the composite nature of Haldane's rule and its genetic bases. The American Naturalist 142:187-212. DOI: https://doi.org/10.1086/285534, PMID: 1 9425975

Zhang L, Sun T, Woldesellassie F, Xiao H, Tao Y. 2015. Sex ratio meiotic drive as a plausible evolutionary mechanism for hybrid male sterility. PLOS Genetics 11:e1005073. DOI: https://doi.org/10.1371/journal.pgen. 1005073, PMID: 25822261 


\section{Appendix 1}

\section{Performance of $\boldsymbol{G}_{\min }$ - detecting introgression from population genomic data}

Using the $G_{\min }$ statistic (Geneva et al., 2015), we report three findings in the main text regarding historical gene flow between Drosophila simulans and D. mauritiana:

1. $1.9 \%$ of $10-\mathrm{kb}$ windows show evidence for recent introgression between these species;

2. recent introgression is significantly underrepresented on the $\mathrm{X}$ chromosome relative to the autosomes; and

3. the lone X-linked region identified as recently introgressed between species contains the previously characterized meiotic drive loci, Dox and MDox.

In this appendix, we present analyses, simulations, and arguments that support these inferences.

To test $G_{\min }$ 's power to detect introgression between $D$. simulans and $D$. mauritiana, we used msmove simulations similar to those described in the Methods, assuming a population size $N_{e}$ of 1,000,000 and 10 generations per year. We simulated divergence followed by gene flow at three times in the past: 400, 4000 and 40,000 years ago. Levels of simulated gene flow were tuned to approximate those observed genome-wide in our data (ms migration probability of 0.008 , corresponding to migration occurring in $2-4 \%$ of $10-\mathrm{kb}$ windows). Each simulation modeled divergence and gene flow within a $10-\mathrm{kb}$ segment using empirical estimates of population mutation and recombination rate parameters estimated from each 10 $\mathrm{kb}$ window in our data. $G_{\min }$ was then calculated for each simulated window.

We then used the same procedure described in the Methods to evaluate whether the simulated value of $G_{\min }$ for a given window was an outlier by performing 10,000 msmove simulations without gene flow and comparing the $G_{\min }$ value from the gene flow simulation to the distribution of $G_{\min }$ values from the strictly allopatric simulations. Windows were deemed to be $G_{\min }$ outliers if they fell in the lowest 0.001 quantile of the non-gene flow simulated distribution. We repeated these steps 100 times for each 10-kb window at each of the three gene flow time points. The power of $G_{\min }$ was determined by measuring the concordance between windows identified as outliers by our procedure and windows that actually contained a simulated gene flow event.

\section{$\mathbf{G}_{\min }$ identifies recent introgression}

The properties and behavior of the $G_{\min }$ statistic have been explored in several previous publications that used coalescent simulations to explore a range of mutation, recombination, and migration parameters. These analyses determined that $G_{\min }$ statistical power is robust to variation in recombination and mutation rates (Geneva et al., 2015; Rosenzweig et al., 2016; Schrider et al., 2018). For no set of mutation or recombination parameters considered does $G_{\min }$ produce an unacceptably high rate of false positives. $G_{\min }$ power is however dependent on the timing of introgression (Geneva et al., 2015; Rosenzweig et al., 2016; Schrider et al., 2018), as shown by simulations that assume levels of gene flow comparable to those observed in our data (Table 1). We find that $G_{\text {min }}$ detects $86 \%, 41 \%$, and $2 \%$ of simulated gene flow events that occurred 400 years, 4,000 years, and 40,000 years ago, respectively (Table 1). While the false positive rate is higher for simulated gene flow that occurred 40,000 years ago, the total number of $10-k b$ windows with significant $G_{\min }$ values is very small; consequently, the total number of false positives is very small as well. We therefore conclude that $G_{\min }$ may be unreliable for older introgressions but identifies younger introgressions with high confidence. 
Appendix 1-table $1 G_{\min }$ and power to detect simulated introgression on the $\mathrm{X}$ chromosome and autosomes. Numbers in parentheses indicate the standard deviation from 100 replicate simulations

\begin{tabular}{|c|c|c|c|c|c|c|}
\hline & \multicolumn{2}{|l|}{400 ybp } & \multicolumn{2}{|l|}{4000 ybp } & \multicolumn{2}{|c|}{40,000 ybp } \\
\hline & A & $x$ & $\mathbf{A}$ & $x$ & A & $x$ \\
\hline $\begin{array}{l}\text { Windows with migration } \\
\text { (\#) }\end{array}$ & $\begin{array}{l}202.12 \\
(18)\end{array}$ & $31.06(7.2)$ & $\begin{array}{l}250.65 \\
(16)\end{array}$ & $66.48(10)$ & $281.15(20)$ & $65.22(8.7)$ \\
\hline $\begin{array}{l}\text { Windows with migration } \\
\text { (\%) }\end{array}$ & $\begin{array}{l}2.4 \% \\
(0.21)\end{array}$ & $1.7 \%(0.4)$ & $3 \%(0.19)$ & $\begin{array}{l}3.7 \% \\
(0.56)\end{array}$ & $3.4 \%(0.24)$ & $3.6 \%(0.48)$ \\
\hline $\begin{array}{l}\text { Significant } G_{\min } \text { windows } \\
(\#)\end{array}$ & $\begin{array}{l}179.08 \\
(17)\end{array}$ & $28.5(6.4)$ & $\begin{array}{l}111.44 \\
(9.8)\end{array}$ & $27.02(4.7)$ & $15.87(4.4)$ & $\begin{array}{l}2.4 \\
(1.3)\end{array}$ \\
\hline $\begin{array}{l}\text { Significant } G_{\min } \text { windows } \\
(\%)\end{array}$ & $2.2 \%(0.2)$ & $\begin{array}{l}1.6 \% \\
(0.35)\end{array}$ & $\begin{array}{l}1.3 \% \\
(0.12)\end{array}$ & $\begin{array}{l}1.5 \% \\
(0.26)\end{array}$ & $\begin{array}{l}0.19 \% \\
(0.052)\end{array}$ & $\begin{array}{l}0.13 \% \\
(0.07)\end{array}$ \\
\hline True positive rate & $96 \%(1.5)$ & $95 \%(3.9)$ & $94 \%(2.4)$ & $93 \%(5.1)$ & $45 \%(11)$ & $30 \%(34)$ \\
\hline False postive rate & $3.7 \%(1.5)$ & $4.8 \%(3.9)$ & $5.7 \%(2.4)$ & $6.8 \%(5.1)$ & $55 \%(11)$ & $70 \%(34)$ \\
\hline Migration Events Detected & $85 \%(3.2)$ & $88 \%(7.5)$ & $42 \%(2.9)$ & $38 \%(5.1)$ & $\begin{array}{l}2.6 \% \\
(1)\end{array}$ & $1.2 \%(1.4)$ \\
\hline
\end{tabular}

DOI: https://doi.org/10.7554/eLife.35468.034

\section{Comparing introgression on the $X$ versus autosomes}

$X$-linked loci have (generally) smaller effective population sizes than autosomal loci and hence lower levels of nucleotide polymorphism. We tested if the systematically lower observed polymorphism on the $X$ can explain its lower levels of introgression detected by $G_{\min }$ using coalescent simulations, resampling, and additional statistical analyses of our empirical results.

First, we note that $G_{\min }$ statistical significance for each $10-\mathrm{kb}$ window was determined by Monte Carlo simulation of neutral genealogies derived from two populations diverging in allopatry (no gene flow). As these simulations used estimates of $\theta$ and $\rho$ drawn from each 10$\mathrm{kb}$ window, they necessarily incorporate systematic differences between the $\mathrm{X}$ and autosomes in these parameters when generating $P$-values. Second, the simulations presented in Table 1 show no significant difference in power (true positive rate or proportion of migration events detected) between X-linked and autosomal windows. Third, using a resampling approach, we generated 10,000 'X-matched autosome' datasets, each drawn randomly from autosomal $10-\mathrm{kb}$ windows, that closely matched the distribution of polymorphism among true $X$-linked windows and tallied the number of significant $G_{\min }$ windows (Figure 1). For 10,000 'X-matched autosome' datasets matching $X$-linked polymorphism in $D$. simulans, no dataset had as few or fewer significant $G_{\text {min }}$ windows than the actual X-linked data ( $p<0.0001)$; for 10,000 'X-matched autosome' datasets matching polymorphism in $D$. mauritiana, only one dataset had as few significant $G_{\text {min }}$ windows as the actual $X$-linked data $(p=0.0001)$. These findings suggest that the observed paucity of introgressions on the $\mathrm{X}$ chromosome cannot be explained simply by its lower levels of polymorphism.

Our data do reveal negative correlations between the $G_{\min } P$-value and polymorphism within D. simulans (Spearman's $\rho=-0.22, \mathrm{p}<0.0001)$ and within $D$. mauritiana $(\rho=-0.38$, $\mathrm{p}<0.0001$ ). Importantly, this correlation is driven by variation in $P$-values among the large majority of non-significant windows (see Figure 5-figure supplement 2). However, significant $G_{\min }$ windows on average do have different levels of polymorphism than nonsignificant ones. In D. simulans, significant $G_{\min }$ windows have less polymorphism than nonsignificant windows, whereas, in $D$. mauritiana, significant $G_{\min }$ windows have more polymorphism than non-significant windows (Figure 5-figure supplement 2; Wilcoxon test $p<0.0001$ for both species). The observation that significant $G_{\min }$ windows have elevated polymorphism in $D$. mauritiana may reflect the direction of gene flow: the presence of 
foreign alleles will tend to elevate diversity in the receiving ( $D$. mauritiana) population but not the donor population (D. simulans).

\section{Patterson's D statistic may not be appropriate for $\mathrm{X}$-autosome comparisons}

In the main text, we report that Patterson's $D$ for the genome $(D=0.0812$, combining all chromosomes), is smaller than that for the the $X$ chromosome $(D=0.1054$, excluding the $130-\mathrm{kb}$ Dox region). For all autosomes combined, $D=0.077$, yielding a $\mathrm{X} / \mathrm{A}$ ratio of $D=1.361$. Superficially, these values could imply the possibility of more introgression on the $X$ than the autosomes and would therefore seem to contradict the $G_{\min }$ results which suggest the opposite. We suggest however that Patterson's $D$ statistic may be inappropriate for simple $\mathrm{X}$ versus autosome comparisons and that discrepancies between Patterson's $D$ and scans for introgression are not unique to our study.

In the case of constant population size, the expected value of $D$ is inversely related to $N_{e}$ (Green et al., 2010; Durand et al., 2011). As a result, under most circumstances, a larger value of $D$ is expected for the $X$ chromosome even if all else- including the degree of introgression - is constant. To illustrate the point, we calculated expected $D$ using standard assumptions for our Drosophila species and obtain values similar to those estimated from the data. The simplifying assumptions for all calculations are:

- constant $N_{e}=1,000,000$ for all species

- 10 generations per year

- a three-species polytomy of D. mauritiana, D. simulans, D. sechellia

- a speciation time $=250,000$ years $(2,500,000$ gens $)$ in the past

- a single pulse of gene flow occurring 50,000 years (500,000 gens) in the past

- introgression probability, $f=0.05$

Using these assumptions and Equation 5 from (Durand et al., 2011) yields $E[D]=0.072$. Taking this value as a plausible autosomal expectation for $D$, we considered three different $N_{\mathrm{e}}$ values for the $\mathrm{X}$, while holding all other parameters constant. Table 2 provides expectations for $D$ on the $X$ chromosome and $X / A$ ratios of $D$.

Appendix 1-table 2. X chromosome, and X/A ratio, for expectation of Patterson's D.

\begin{tabular}{llll}
$\mathbf{X} / \mathbf{A}$ ratio of $\mathbf{N}_{\mathbf{e}}$ & Rationale & $\mathbf{E}[\mathbf{D}]$ & X/A ratio of $\boldsymbol{D}$ \\
\hline $\mathbf{0 . 7 5}$ & $1: 1$ sex ratio, random mating, etc. & 0.094 & 1.309 \\
\hline $\mathbf{0 . 6 5 6}$ & Observed X/A nucleotide diversity in D. mauritiana & 0.106 & 1.479 \\
\hline $\mathbf{0 . 7 7 8}$ & Observed X/A nucleotide diversity in D. simulans & 0.091 & 1.265
\end{tabular}

DOI: https://doi.org/10.7554/eLife.35468.035

For all three cases, the $X / A$ ratio of $E[D]$ is greater than one and comparable to the ratio estimated from our data $(X / A$ ratio $=1.361)$ despite no difference in assumptions about the amount or timing of gene flow between the $\mathrm{X}$ and autosomes.

Notably, discrepancies between Patterson's $D$ and focused introgression scans are not unique to our study. Green et al. (Green et al., 2010) developed the D statistic and estimated $D$ between Neanderthal and non-African humans for all 23 chromosomes (their Supplementary Table S47). Between Asians and Neanderthals, $D$ is 2.3-fold higher for the $X$ chromosome than the mean of the 22 autosomes (Table S47), which would seem to imply a greater rate of introgression on the X. Later work by the same group (Sankararaman et al., 2014; Sankararaman et al., 2016) scanned genomes for introgression using relative sequence distances and haplotype length as criteria and found a significant dearth of introgression on the $X$ relative to the autosomes (X/A introgression $\sim 20 \%$ ). Thus, paralleling our results, Patterson's $D$ between Asians and Neanderthals implies excess gene flow on the $X$, whereas the genomic scan implies the opposite. As expectations for Patterson's $D$ on the 
$X$ versus the autosomes are confounded by effective population size, it seems imprudent to draw strong conclusions about relative gene flow from the $D$ statistic alone.

A
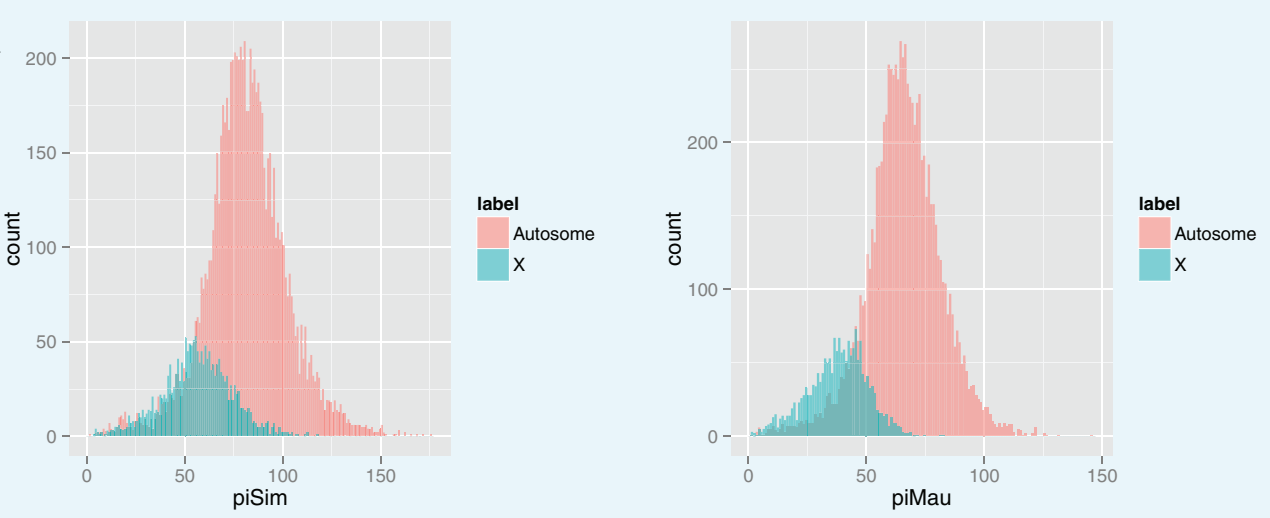

B
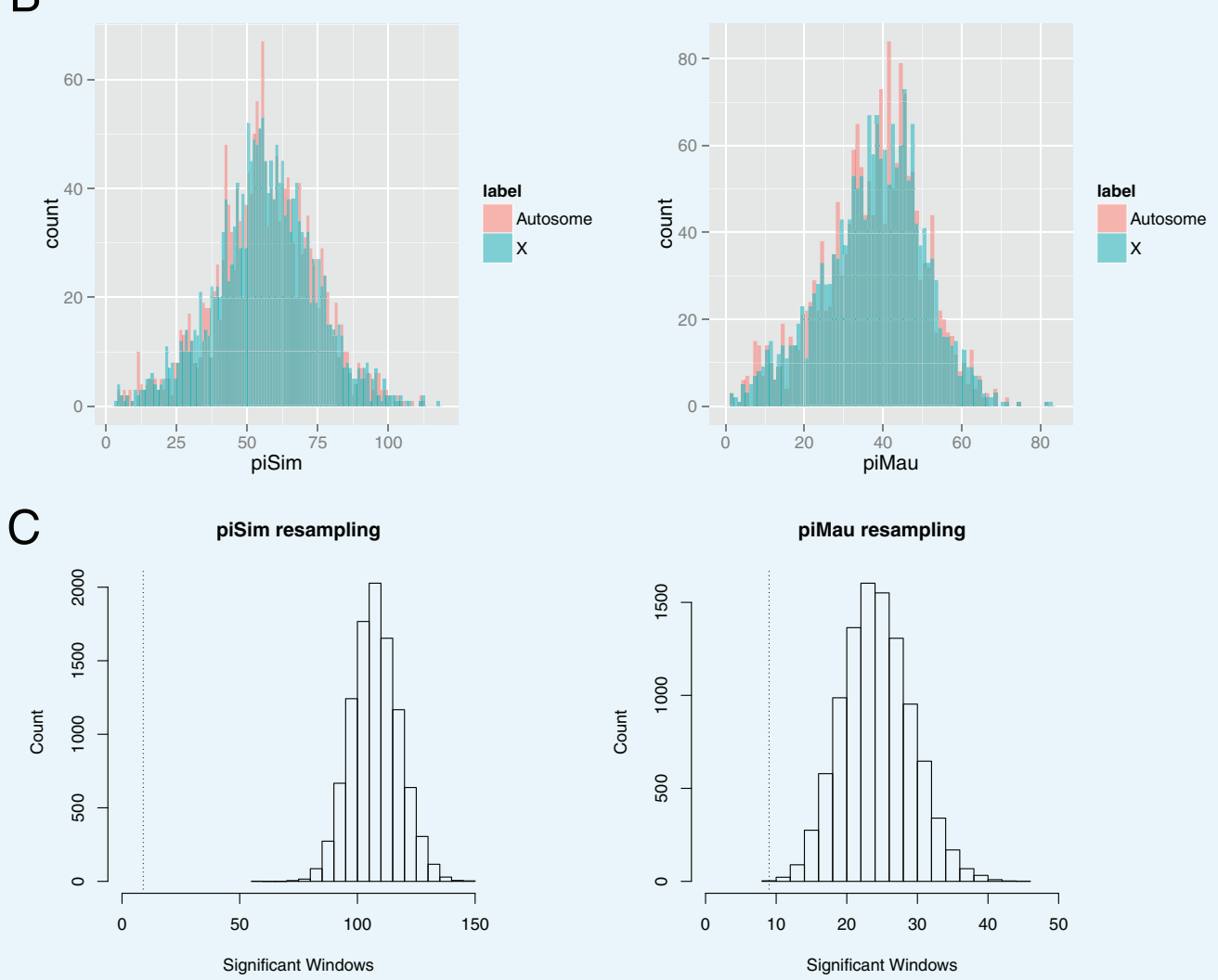

Appendix 1-figure 1 Resampled autosomal 10-kb windows matching X-chromosome polymorphism. (A) Distributions of polymorphism within $10-\mathrm{kb}$ windows for the $\mathrm{X}$ chromosome and autosomes in D. simulans and D. mauritiana. (B) Exemplar resampled autosomal data sets matching $\mathrm{X}$-chromosome polymorphism for $D$. simulans and D. mauritiana. (C) Distribution of the number of resampled windows with significant $G_{\min }$ values across 10,000 replicate resampled data sets. Vertical dotted lines indicate the observed number of significant $X$-linked windows in each species.

DOl: https://doi.org/10.7554/eLife.35468.036 